\title{
Directed percolation process in the presence of velocity fluctuations: Effect of compressibility and finite correlation time
}

\author{
N. V. Antonov, ${ }^{1}$ M. Hnatič,,${ }^{2,3}{ }^{3}$ A. S. Kapustin, ${ }^{1}$ T. Lučivjanský,,${ }^{3,5}$ and L. Mižišin ${ }^{3}$ \\ ${ }^{1}$ Department of Theoretical Physics, St. Petersburg University, \\ Ulyanovskaya 1, St. Petersburg, Petrodvorets, 198504 Russia \\ ${ }^{2}$ Institute of Experimental Physics, SAS, 04001 Košice, Slovakia \\ ${ }^{3}$ Faculty of Sciences, P.J. Šafarik University, 04154 Košice, Slovakia \\ ${ }^{4}$ Bogoliubov Laboratory of Theoretical Physics, JINR, 141980 Dubna, Moscow Region, Russia \\ ${ }^{5}$ Fakultät für Physik, Universität Duisburg-Essen, D-47048 Duisburg, Germany
}

(Dated: May 24, 2022)

\begin{abstract}
The direct bond percolation process (Gribov process) is studied in the presence of random velocity fluctuations generated by the Gaussian self-similar ensemble with finite correlation time. We employ the renormalization group in order to analyze a combined effect of the compressibility and finite correlation time on the long-time behavior of the phase transition between an active and an absorbing state. The renormalization procedure is performed to the one-loop order. Stable fixed points of the renormalization group and their regions of stability are calculated in the one-loop approximation within the three-parameter $(\varepsilon, y, \eta)$-expansion. Different regimes corresponding to the rapid-change limit and frozen velocity field are discussed, and their fixed points' structure is determined in numerical fashion.
\end{abstract}

\section{INTRODUCTION}

The non-equilibrium physical systems constitute an exciting research topic to which a lot of effort has been made during last decades [1,3]. Absorbing phase transitions between active (fluctuating) and inactive (absorbing) states are of particular importance. In these transitions large scale spatio-temporal fluctuations of an underlying order parameter take place and the resulting collective behavior is similar to equilibrium phase transitions. Such behavior could be observed in many natural phenomena ranging from physics, chemistry, biology, economy or even sociology.

A fundamental part of this type of systems belongs to the directed percolation (DP) universality class [2, 4]. As pointed by Janssen and Grassberger [5, 6], necessary conditions are: i) a unique absorbing state, ii) short-ranged interactions, iii) a positive order parameter and iv) no extra symmetry or additional slow variables. Among a few models described within this framework we name population dynamics, reaction-diffusion problems [7], percolation processes [8], hadron interactions [9], etc. These models are usually considered without an inclusion of additional interactions within the mode-mode coupling approach [10]. However, in realistic situations impurities and defects, which are not taken into account in the original DP formulation, are expected to cause a change in the universal properties of the model. This is believed to be one of the reasons why there are not so many direct experimental realizations [11, 12] of the percolation process itself. A study of deviations from the ideal situation could proceed in different routes and this still constitutes a topic of an ongoing debate [2. A substantial effort has been made in studying a long-range interaction using Lévy-flight jumps [13 15], effects of immunization [8, 16], mutations [17, feedback of the environment on the percolating density [18, or in the presence of spatially quenched disorder [19]. In general, the novel behavior is observed with a possibility that critical behavior is lost. For example, the presence of a quenched disorder in the latter case causes a shift of the critical fixed point to the unphysical region. This leads to such interesting phenomena as an activated dynamical scaling or Griffiths singularities 20 23].

In this paper, we focus on the directed bond percolation process in the presence of advective velocity fluctuations. Velocity fluctuations are hardly avoidable in any of experiments. For example, a vast majority of chemical reactions occurs at finite temperature, which is inevitably encompassed with the presence of a thermal noise. Furthermore, disease spreading and chemical reactions could be affected by the turbulent advection to a great extent. Fluid dynamics is in general described by the Navier-Stokes equations 24]. A general solution of these equations remains an open question [25, 26. However, to provide more insight we restrict ourselves to a more decent problem. Namely, we assume that the velocity field is given by the Gaussian velocity ensemble with prescribed statistical properties [27, 28]. Although this assumption appears as oversimplified, compared to the realistic flows at the first sight, it nevertheless captures essential physical information about advection processes [27, 29, 30].

Recently, there has been increased interest in different advection problems in compressible turbulent flows [31 34]. These studies show that compressibility plays a decisive role for population dynamics or chaotic mixing of colloids. Our main aim is to investigate an influence of compressibility 35, 36 on the critical properties of the directed bond percolation process 2. To this end, the advective field is described by the Kraichnan model with finite correlation time, in which not only a solenoidal (incompressible) but also a potential (compressible) part of the velocity statistics is involved. Note that in our model 
there is no backward influence of percolating field on the velocity fluctuations. In other words, our model corresponds to the passive advection of the reacting scalar field.

A powerful tool for analysis of the critical behavior is the renormalization group ( $\mathrm{RG}$ ) [37 39] method. It constitutes a theoretical framework which allows one to compute universal quantities in a controllable manner and also to determine universality classes of the physical system. Here this method is employed in order to determine the scaling behavior in the vicinity of the phase transition between the active and absorbing state with an emphasis on a possible type of critical behavior.

The remainder of the paper proceeds as follows. In Sec. [II] we introduce a coarse-grained formulation of the problem, which we reformulate into the field-theoretic model. In Sec. III, we describe the main steps of the perturbative RG procedure. In Sec. IV] we present analysis of possible regimes involved in the model. We analyze numerically and to some extent analytically fixed points' structure. In Sec. V] we give a concluding summary. Technical details concerning calculation of RG constants and functions are presented in Appendix A and Appendix B. The coordinates of all fixed points are given in Appendix C.

\section{THE MODEL}

A continuum description of DP in terms of a density $\psi=\psi(t, \boldsymbol{x})$ of infected individuals typically arises from a coarse-graining procedure in which a large number of fast microscopic degrees of freedom are averaged out. A loss of the physical information is supplemented by a Gaussian noise in a resulting Langevin equation. Obviously, a correct mathematical description has to be in conformity regarding the absorbing state condition: $\psi=0$ is always a stationary state and no microscopic fluctuation can change that. The coarse grained stochastic equation then reads 8

$$
\partial_{t} \psi=D_{0}\left(\nabla^{2}-\tau_{0}\right) \psi-\frac{g_{0} D_{0}}{2} \psi^{2}+\xi,
$$

where $\xi$ denotes the noise term, $\partial_{t}=\partial / \partial t$ is the time derivative, $\nabla^{2}$ is the Laplace operator, $D_{0}$ is the diffusion constant, $g_{0}$ is the coupling constant and $\tau_{0}$ measures a deviation from the threshold value for injected probability. It can be thought as an analog to the temperature variable in the standard $\varphi^{4}$-theory [8, 38]. Due to dimensional reasons, we have extracted the dimensional part from the interaction term (See later Sec. IIIA). Here and henceforth we distinguish between unrenormalized (with the subscript " 0 ") quantities and renormalized terms (without the subscript "0"). The renormalized fields will be later denoted by the subscript $R$.

It can be rigorously proven [5] that the Langevin equation (1) captures the gross properties of the percolation process and contains essential physical information about the large-scale behavior of the non-equilibrium phase transition between the active $(\psi>0)$ and the absorbing state $(\psi=0)$. The Gaussian noise term $\xi$ with zero mean has to satisfy the absorbing state condition. Its correlation function can be chosen in the following form

$\left\langle\xi\left(t_{1}, \boldsymbol{x}_{1}\right) \xi\left(t_{2}, \boldsymbol{x}_{2}\right)\right\rangle=g_{0} D_{0} \psi\left(t_{1}, \boldsymbol{x}_{1}\right) \delta\left(t_{1}-t_{2}\right) \delta^{(d)}\left(\boldsymbol{x}_{1}-\boldsymbol{x}_{2}\right)$

up to irrelevant contributions [3. Here $\delta^{(d)}(\boldsymbol{x})$ is the $d$-dimensional generalization of the standard Dirac $\delta(x)$ function.

A further step consists in incorporating of the velocity fluctuations into the model (1). The standard route [24] based on the replacement $\partial_{t}$ by the Lagrangian derivative $\partial_{t}+(\boldsymbol{v} \cdot \boldsymbol{\nabla})$ is not sufficient due to the assumed compressibility. As shown in [40, the following replacement is then adequate

$$
\partial_{t} \rightarrow \partial_{t}+(\boldsymbol{v} \cdot \boldsymbol{\nabla})+a_{0}(\boldsymbol{\nabla} \cdot \boldsymbol{v}),
$$

where $a_{0}$ is an additional positive parameter, whose significance will be discussed later. Note that the last term in (3) contains a divergence of the velocity field $\boldsymbol{v}$ and thus $\boldsymbol{\nabla}$ operator does not act on what could possibly follow.

Following [36], we consider the velocity field to be a random Gaussian variable with zero mean and a translationally invariant correlator given as follows:

$$
\left\langle v_{i}(t, \boldsymbol{x}) v_{j}(0, \mathbf{0})\right\rangle=\int \frac{\mathrm{d} \omega}{2 \pi} \int \frac{\mathrm{d}^{d} \boldsymbol{k}}{(2 \pi)^{d}} D_{v}(\omega, \boldsymbol{k}) \mathrm{e}^{-i \omega t+\boldsymbol{k} \cdot \boldsymbol{x}},
$$

where the kernel function $D_{v}(\omega, \boldsymbol{k})$ takes the form

$$
D_{v}(\omega, \boldsymbol{k})=\left[P_{i j}^{k}+\alpha Q_{i j}^{k}\right] \frac{g_{10} u_{10} D_{0}^{3} k^{4-d-y-\eta}}{\omega^{2}+u_{10}^{2} D_{0}^{2}\left(k^{2-\eta}\right)^{2}} .
$$

Here, $P_{i j}^{k}=\delta_{i j}-k_{i} k_{j} / k^{2}$ is a transverse and $Q_{i j}^{k}=$ $k_{i} k_{j} / k^{2}$ a longitudinal projection operator, $k=|\boldsymbol{k}|$, and $d$ is the dimensionality of the $\boldsymbol{x}$ space. A positive parameter $\alpha>0$ can be interpreted as the simplest possible deviation 35] from the incompressibility condition $\boldsymbol{\nabla} \cdot \boldsymbol{v}=0$. The incompressible case, $\alpha=0$, was analyzed in previous works [40 44]. The coupling constant $g_{10}$ and the exponent $y$ describe the equal-time velocity correlator or, equivalently, the energy spectrum [25, 28, 36 . of the velocity fluctuations. The constant $u_{10}>0$ and the exponent $\eta$ are related to the characteristic frequency $\omega \simeq u_{10} D_{0} k^{2-\eta}$ of the mode with the wavelength $k$.

The momentum integral in (4) has an infrared (IR) cutoff at $k=m$, where $m \sim 1 / \bar{L}$ is the reciprocal of the integral scale $L$. A precise form of the cutoff [30, 45] is actually unimportant and its role is to provide us with IR regularization. Further, dimensional considerations show that the bare coupling constants $g_{10}$ and $u_{10}$ are related to the characteristic UV momentum scale $\Lambda$ by

$$
g_{10} \simeq \Lambda^{y}, \quad u_{10} \simeq \Lambda^{\eta} .
$$


The choice $y=8 / 3$ gives the famous Kolmogorov "fivethirds" law for the spatial velocity correlations, and $\eta=$ $4 / 3$ corresponds to the Kolmogorov frequency [25].

The exponents $y$ and $\eta$ are analogous to the standard expansion parameter $\varepsilon=4-d$ in the static critical phenomena. It can be shown that the upper critical dimension of the pure percolation problem [8] is also $d_{c}=4$. Therefore, we retain the standard notation for the exponent $\varepsilon$. According to the general rules 39] of the RG approach, we formally assume that the exponents $\varepsilon, y$ and $\eta$ are of the same order of magnitude and constitute small expansion parameters of perturbation theory.

The kernel function in 5 is chosen in a quite general form and as such it contains various special limits. They simplify numerical analysis of the resulting equations and allows us to gain a deeper physical insight into the model. Possible limiting cases are

i) The rapid-change model, which corresponds to the limit $u_{10} \rightarrow \infty, g_{10}^{\prime} \equiv g_{10} / u_{10}=$ const. Then for the kernel function we have

$$
D_{v}(\omega, \boldsymbol{k}) \propto g_{10}^{\prime} D_{0} k^{-d-y+\eta}
$$

and obviously the velocity correlator is $\delta$-correlated in a time variable.

ii) The frozen velocity field, which arises in the limit $u_{10} \rightarrow 0$ and the kernel function corresponds to

$$
D_{v}(\omega, \boldsymbol{k}) \propto g_{0} D_{0}^{2} \pi \delta(\omega) k^{2-d-y} .
$$

iii) The purely potential velocity field, which is obtained for $\alpha \rightarrow \infty$ with $\alpha g_{10}=$ constant. This limit is similar to the model of random walks in a random environment with long-range correlations [46, 47].

iv) The turbulent advection, for which the $y=2 \eta=$ $8 / 3$. This choice mimics properties of the genuine turbulence and leads to the celebrated Kolmogorov scaling [25].

For an effective use of the RG method it is advantageous to rewrite the stochastic problem (1.5) into the field-theoretic formulation. This could be achieved in the standard fashion [48 50] and the resulting dynamic functional is

$$
\mathcal{S}[\varphi]=\mathcal{S}_{\text {diff }}[\varphi]+\mathcal{S}_{\text {vel }}[\varphi]+\mathcal{S}_{\text {int }}[\varphi],
$$

where $\varphi=\{\tilde{\psi}, \psi, \boldsymbol{v}\}$ stands for the complete set of fields and $\tilde{\psi}$ is the auxiliary (Martin-Siggia-Rose) response field [51. The first term represents a free part of the equation (1) and is given by the following expression:

$$
\mathcal{S}_{\mathrm{diff}}[\varphi]=\int \mathrm{d} t \int \mathrm{d}^{d} \boldsymbol{x}\left\{\tilde{\psi}\left[\partial_{t}-D_{0} \nabla^{2}+D_{0} \tau_{0}\right] \psi\right\} .
$$

Since the velocity fluctuations are governed by the Gaussian statistics, the corresponding averaging procedure is performed with the quadratic functional

$$
\mathcal{S}_{\text {vel }}[\boldsymbol{v}]=\frac{1}{2} \int \mathrm{d} t_{1} \int \mathrm{d} t_{2} \int \mathrm{d}^{d} \boldsymbol{x}_{1} \int \mathrm{d}^{d} \boldsymbol{x}_{2} \boldsymbol{v}_{i}\left(t_{1}, x_{1}\right)
$$

$$
D_{i j}^{-1}\left(t_{1}-t_{2}, \boldsymbol{x}_{1}-\boldsymbol{x}_{2}\right) \boldsymbol{v}_{j}\left(t_{2}, \boldsymbol{x}_{2}\right),
$$

where $D_{i j}^{-1}$ is the kernel of the inverse linear operation in (4). The final interaction part can be written as

$$
\begin{aligned}
\mathcal{S}_{\text {int }}[\varphi] & =\int \mathrm{d} t \int \mathrm{d}^{d} \boldsymbol{x}\left\{\frac{D_{0} \lambda_{0}}{2}[\psi-\tilde{\psi}] \tilde{\psi} \psi-\frac{u_{20}}{2 D_{0}} \tilde{\psi} \psi \boldsymbol{v}^{2}\right. \\
& \left.+\tilde{\psi}(\boldsymbol{v} \cdot \boldsymbol{\nabla}) \psi+a_{0} \tilde{\psi}(\boldsymbol{\nabla} \cdot \boldsymbol{v}) \psi\right\} .
\end{aligned}
$$

All but the third term in 12 directly stem from the nonlinear terms in (1) and (3). The third term proportional to $\propto \tilde{\psi} \psi \boldsymbol{v}^{2}$ deserves a special consideration. The presence of this term is prohibited in the original Kraichnan model due to the underlying Galilean invariance. However, in our case the general form of the velocity kernel function does not lead to such restriction. Moreover, by direct inspection of the perturbative expansion, one can show that this kind of term is indeed generated under RG transformation (consider second Feynman graph in the expression (A5)). This term was considered for the first time in our previous work [44, where the incompressible case is analyzed.

Let us also note that for the linear advection-diffusion equation [24, 36], the choice $a_{0}=1$ corresponds to the conserved quantity $\psi$ (advection of a density field), whereas for the choice $a_{0}=0$ the conserved quantity is $\tilde{\psi}$ (advection of a tracer field). From the point of view of the renormalization group, the introduction of $a_{0}$ is necessary, because it ensures multiplicative renormalizability of the model [40].

In principle, basic ingredients of any stochastic theory, correlation and response functions of the concentration field $\psi(t, \boldsymbol{x})$, can be computed as functional averages with respect to the weight functional $\exp (-\mathcal{S})$ with action $(9$ ). Further, the field-theoretic formulation summarized in (10)- 12 has an additional advantage to be amenable to the full machinery of (quantum) field theory [38, 39]. In the subsequent section, we apply the RG perturbative technique [39] that allows us to study the model in the vicinity of its upper critical dimension $d_{c}=4$.

\section{RENORMALIZATION GROUP ANALYSIS}

An important goal of statistical theories is the determination of correlation and response functions (usually called Green functions) of the dynamical fields as functions of the space-time coordinates. Traditionally, these functions are represented in the form of sums over the Feynman diagrams 38, 39. The functional formulation provides a convenient theoretical framework suitable for applying methods of quantum field theory. Using the RG method [38, 52] it is possible to determine the infrared (IR) asymptotic (large spatial and time scales) behavior of the correlation functions. A proper renormalization procedure is needed for the elimination of ultraviolet (UV) divergences. There are various renormalization prescriptions applicable for such problem, each 
with its own advantages [38. In this work, we employ the minimal subtraction (MS) scheme. UV divergences manifest themselves in the form of poles in the small expansion parameters, and the minimal subtraction scheme is characterized by discarding all finite parts of the Feynman graphs in the calculation of the renormalization constants. In the vicinity of critical points large fluctuations on all spatio-temporal scales dominate the behavior of the system, which in turn results in the divergences in the Feynman graphs. The resulting RG functions satisfy certain differential equations and their analysis provides us with an efficient computational technique for estimation of universal quantities.

\section{A. Canonical dimensions}

In order to apply the dimensional regularization for evaluation of renormalization constants, an analysis of possible superficial divergences has to be performed. For translationally invariant systems, it is sufficient to analyze only 1-particle irreducible (1PI) graphs [37, 38. In contrast to static models, dynamic models [3, 39] contain two independent scales: a frequency scale $d_{Q}^{\omega}$ and a momentum scale $d_{Q}^{k}$ for each quantity $Q$. The corresponding dimensions are found using the standard normalization conditions

$$
\begin{array}{ll}
d_{k}^{k}=-d_{x}^{k}=1, & d_{\omega}^{k}=d_{t}^{k}=0, \\
d_{k}^{\omega}=d_{x}^{\omega}=0, & d_{\omega}^{\omega}=-d_{t}^{\omega}=1
\end{array}
$$

together with a condition for field-theoretic action to be a dimensionless quantity. Using the quantities $d_{Q}^{\omega}$ and $d_{Q}^{k}$, the total canonical dimension $d_{Q}$,

$$
d_{Q}=d_{Q}^{k}+2 d_{Q}^{\omega}
$$

can be introduced, whose precise form is obtained from a comparison of the IR most relevant terms $\left(\partial_{t} \propto \nabla^{2}\right)$ in the action 10 . The total dimension $d_{Q}$ for the dynamical models plays the same role as the conventional (momentum) dimension does in static problems. The dimensions of all quantities for the model are summarized in Table I. It follows that the model is logarithmic (when coupling constants are dimensionless) at $\varepsilon=y=\eta=0$, and the UV divergences are in principle realized as poles in these parameters. The total canonical dimension of an arbitrary 1 - irreducible Green function is given by the relation

$$
d_{\Gamma}=d_{\Gamma}^{k}+2 d_{\Gamma}^{\omega}=d+2-\sum_{\varphi} N_{\varphi} d_{\varphi}, \quad \varphi \in\{\tilde{\psi}, \psi, \boldsymbol{v}\}
$$

The total dimension $d_{\Gamma}$ in the logarithmic theory is the formal degree of the UV divergence $\delta_{\Gamma}=\left.d_{\Gamma}\right|_{\varepsilon=y=\eta=0}$. Superficial UV divergences, whose removal requires counterterms, could be present only in those functions $\Gamma$ for which $\delta_{\Gamma}$ is a non-negative integer [39].

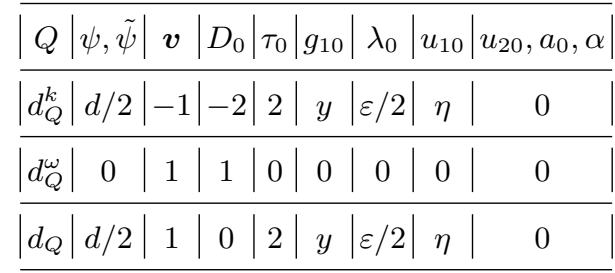

Table I. Canonical dimensions of the bare fields and bare parameters for the model $10-12$.

\begin{tabular}{|c|c|c|c|c|c|}
\hline$\left|\Gamma_{1-i r}\right| \Gamma_{\tilde{\psi} \psi}\left|\Gamma_{\tilde{\psi} \psi \boldsymbol{v}}\right| \Gamma_{\tilde{\psi}^{2} \psi}\left|\Gamma_{\tilde{\psi} \psi^{2}}\right| \Gamma_{\tilde{\psi} \psi \boldsymbol{v}^{2}} \mid$ \\
\hline$d_{\Gamma}$ & 2 & 1 & $\varepsilon / 2$ & $\varepsilon / 2$ & 0 \\
\hline$\delta_{\Gamma}$ & 2 & 1 & 0 & 0 & 0 \\
\hline
\end{tabular}

Table II. Canonical dimensions for the (1PI) divergent Green functions of the model.

Dimensional analysis should be augmented by certain additional considerations. In dynamical models of the type (10)-12, all the 1-irreducible diagrams without the fields $\psi$ vanish, and it is sufficient to consider the functions with $N_{\tilde{\psi}} \geq 1$. As was shown in 40], the rapidity symmetry (24) requires also $N_{\psi} \geq 1$ to hold. Using these considerations together with relation (15), possible UV divergent structures are expected only for the 1PI Green functions listed in Table II.

\section{B. Computation of the RG constants}

In this section, the main steps of the perturbative RG approach are summarized, deferring the explicit results of the RG constants and RG functions (anomalous dimensions and beta functions) to Appendices $\mathrm{A}$ and $\mathrm{B}$.

A starting point of the perturbation theory is a free part of the action given by expressions $(10)$ and (11). By graphical means, they are represented as lines in the Feynman diagrams, whereas the non-linear terms in 12 correspond to vertices connected by the lines.

For the calculation of the RG constants we have employed dimensional regularization in the combination with the MS scheme 38. Since the finite correlated case involves two different dispersion laws: $\omega \propto k^{2}$ for the scalar and $\omega \propto k^{2-\eta}$ for the velocity fields, the calculations for the renormalization constants become rather cumbersome already in the one-loop approximation [28, 36]. However, it was shown 53 that to the twoloop order it is sufficient to consider the choice $\eta=0$. This significantly simplifies practical calculations and as can be seen in (A6), the only poles to the one-loop order are of two types: either $1 / \varepsilon$ or $1 / y$. This simple picture pertains only to the lowest orders in a perturbation scheme. In higher order terms, poles in the form of general linear combinations in $\varepsilon, \eta$ and $y$ are expected to arise. 
The perturbation theory of the model $(9)$ is amenable to the standard Feynman diagrammatic expansion [3739. The inverse matrix of the quadratic part in the actions determines a form of the bare propagators. The propagators are presented in the wave-number-frequency representation, which is for the translationally invariant systems the most convenient way for doing explicit calculations. The bare propagators are easily read off from the Gaussian part of the model given by $(10)$ and (11), respectively. Their graphical representation is depicted in Fig. 1. The corresponding algebraic expressions can be easily read off and in the frequency-momentum representation are given by

$$
\begin{aligned}
& \langle\psi \tilde{\psi}\rangle_{0}=\langle\tilde{\psi} \psi\rangle_{0}^{*}=\frac{1}{-i \omega+D_{0}\left(k^{2}+\tau_{0}\right)}, \\
& \langle\boldsymbol{v} \boldsymbol{v}\rangle_{0}=\left[P_{i j}^{k}+\alpha Q_{i j}^{k}\right] \frac{g_{10} u_{10} D_{0}^{3} k^{4-d-y-\eta}}{\omega^{2}+u_{10}^{2} D_{0}^{2}\left(k^{2-\eta}\right)^{2}}
\end{aligned}
$$

or in the time-momentum representation as

$$
\begin{aligned}
& \langle\psi \tilde{\psi}\rangle_{0}=\theta(t) \exp \left(-D_{0}\left[k^{2}+\tau_{0}\right] t\right), \\
& \langle\tilde{\psi} \psi\rangle_{0}=\theta(-t) \exp \left(D_{0}\left[k^{2}+\tau_{0}\right] t\right), \\
& \langle\boldsymbol{v} \boldsymbol{v}\rangle_{0}=\left[P_{i j}^{k}+\alpha Q_{i j}^{k}\right] \frac{g_{10} D_{0}^{2}}{k^{d+y-2}} \mathrm{e}^{-u_{10} D_{0} k^{2-\eta}|t|},
\end{aligned}
$$

where $\theta(t)$ is the Heaviside step function.

The interaction vertices from the nonlinear part 12 ) describe the fluctuation effects connected with the percolation process itself, advection of the concentration field and the interactions between the velocity components. With every such vertex the following algebraic factor

$$
V_{N}\left(x_{1}, \ldots, x_{N} ; \varphi\right)=\frac{\delta^{N} \mathcal{S}_{\text {int }}[\varphi]}{\delta \varphi\left(x_{1}\right) \ldots \delta \varphi\left(x_{N}\right)}, \quad \varphi \in\{\tilde{\psi}, \psi, \boldsymbol{v}\}
$$

is associated [39]. In our model there are four different interaction vertices, which are graphically depicted in Fig. 2 and Fig. 3 respectively. The corresponding vertex factors are

$$
\begin{aligned}
& V_{\tilde{\psi} \psi \psi}=-V_{\tilde{\psi} \tilde{\psi} \psi}=D_{0} \lambda_{0}, \\
& V_{\tilde{\psi} \psi \boldsymbol{v}}=i k_{j}+i a_{0} q_{j}, \\
& V_{\tilde{\psi} \psi \boldsymbol{v} \boldsymbol{v}}=-\frac{u_{20}}{D_{0}} \delta_{i j} .
\end{aligned}
$$

In the expression for $V_{\tilde{\psi} \psi \boldsymbol{v}}$, we have adopted the following convention: $k_{j}$ is the momentum of the field $\psi$ and $q_{j}$ is the momentum of the velocity field $\boldsymbol{v}$. The presence of the interaction vertex $V_{\tilde{\psi} \psi \boldsymbol{v} v}$ leads to the proliferation of the new Feynman graphs (see Appendix A), which were absent in the previous studies [40, 41, 44].

By direct inspection of the Feynman diagrams one can observe that the real expansion parameter is rather $\lambda_{0}^{2}$ than $\lambda_{0}$. This is a direct consequence of the duality symmetry 8 ] of the action for the pure percolation problem with respect to time inversion

$$
\psi(t, \boldsymbol{x}) \rightarrow-\tilde{\psi}(-t, \boldsymbol{x}), \quad \tilde{\psi}(t, \boldsymbol{x}) \rightarrow-\psi(-t, \boldsymbol{x}) .
$$

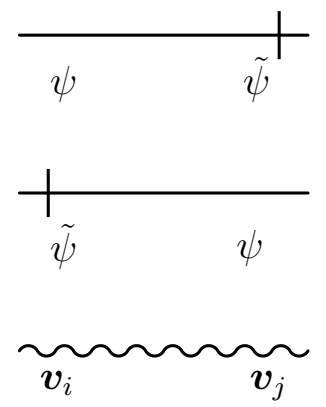

Figure 1. Diagrammatic representation of the bare propagators. The time flows from right to left.

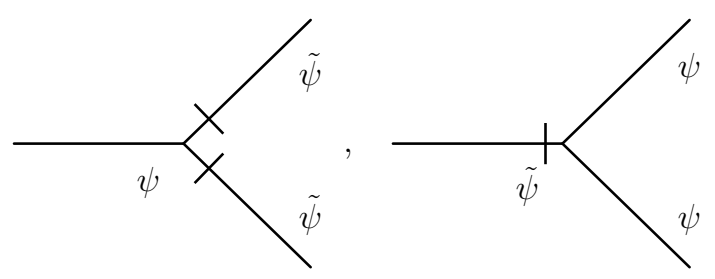

Figure 2. Diagrammatic representation of the interaction vertices describing an ideal directed bond percolation process.

Therefore, we introduce a new charge $g_{20}$ via the relation

$$
g_{20}=\lambda_{0}^{2}
$$

and express the perturbation calculation in terms of this parameter.

In the presence of compressible velocity field the transformation (24) has to be augmented by the transformation

$$
a_{0} \rightarrow 1-a_{0},
$$

as can be easily seen by inserting (24) in 12 and performing integration by parts.

With the help of Table I the renormalized parameters can be introduced in the following manner:

$$
\begin{array}{lll}
D_{0}=D Z_{D}, & \tau_{0}=\tau Z_{\tau}+\tau_{c}, & a_{0}=a Z_{a}, \\
g_{10}=g_{1} \mu^{y} Z_{g_{1}}, & u_{10}=u_{1} \mu^{\eta} Z_{u_{1}}, & \lambda_{0}=\lambda \mu^{\varepsilon / 2} Z_{\lambda}, \\
g_{20}=g_{2} \mu^{\varepsilon} Z_{g_{2}}, & u_{20}=u_{2} Z_{u_{2}}, &
\end{array}
$$

where $\mu$ is the reference mass scale in the MS scheme [38. Note that the term $\tau_{c}$ is a non-perturbative effect [54, 55],

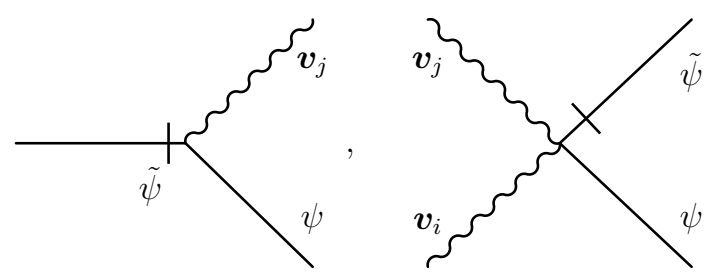

Figure 3. Interaction vertices describing the influence of the advecting velocity field with the order parameter fluctuations. 
which is not captured by the dimensional regularization. The renormalization prescription (27) together with the renormalization of fields

$$
\tilde{\psi}=Z_{\tilde{\psi}} \tilde{\psi}_{R}, \quad \psi=Z_{\psi} \psi_{R}, \quad \boldsymbol{v}=Z_{v} \boldsymbol{v}_{R}
$$

is sufficient for obtaining a fully renormalized theory. Thus, the total renormalized action for the renormalized fields $\varphi_{R} \equiv\left\{\tilde{\psi}_{R}, \psi_{R}, \boldsymbol{v}_{R}\right\}$ can be written in a compact form

$$
\begin{aligned}
\mathcal{S}_{R}\left[\varphi_{R}\right] & =\int \mathrm{d} t \int \mathrm{d}^{d} \boldsymbol{x}\left\{\tilde { \psi } _ { R } \left[Z_{1} \partial_{t}-Z_{2} D \nabla^{2}+Z_{3} D \tau\right.\right. \\
& \left.+Z_{4}\left(\boldsymbol{v}_{R} \cdot \boldsymbol{\nabla}\right)+a Z_{5}\left(\boldsymbol{\nabla} \cdot \boldsymbol{v}_{R}\right)\right] \psi_{R}-\frac{D \lambda}{2}\left[Z_{6} \tilde{\psi}_{R}\right. \\
& \left.\left.-Z_{7} \psi_{R}\right] \tilde{\psi}_{R} \psi_{R}-Z_{8} \frac{u_{2}}{2 D} \tilde{\psi}_{R} \psi_{R} \boldsymbol{v}_{R}^{2}\right\}+ \\
& \frac{1}{2} \int \mathrm{d} t_{1} \int \mathrm{d} t_{2} \int \mathrm{d}^{d} \boldsymbol{x}_{1} \int \mathrm{d}^{d} \boldsymbol{x}_{2} \boldsymbol{v}_{R i}\left(t_{1}, x_{1}\right) \\
& D_{R i j}^{-1}\left(t_{1}-t_{2}, \boldsymbol{x}_{1}-\boldsymbol{x}_{2}\right) \boldsymbol{v}_{R j}\left(t_{2}, \boldsymbol{x}_{2}\right) .
\end{aligned}
$$

The latter term is a renormalized version of (11). The relations between the renormalization constants follow directly from the action (29)

$$
\begin{aligned}
Z_{1} & =Z_{\psi} Z_{\tilde{\psi}}, & Z_{2} & =Z_{\psi} Z_{\tilde{\psi}} Z_{D}, \\
Z_{3} & =Z_{\psi} Z_{\tilde{\psi}} Z_{D} Z_{\tau}, & Z_{4} & =Z_{\psi} Z_{\tilde{\psi}} Z_{v}, \\
Z_{5} & =Z_{\psi} Z_{\tilde{\psi}} Z_{v} Z_{a}, & Z_{6} & =Z_{\psi} Z_{\tilde{\psi}}^{2} Z_{D} Z_{\lambda}, \\
Z_{7} & =Z_{\psi}^{2} Z_{\tilde{\psi}} Z_{D} Z_{\lambda}, & Z_{8} & =Z_{\psi} Z_{\tilde{\psi}} Z_{v}^{2} Z_{u_{2}} Z_{D}^{-1} .
\end{aligned}
$$

The theory is made UV finite through the appropriate choice of the RG constants $Z_{1}, \ldots, Z_{8}$. Afterwards, relations (30) yield the corresponding RG constants for the fields and parameters appearing in relations (27). The explicit results for the RG constants are given in Appendix A.

According to the general rules of the RG method 39, the nonlocal term in action 29 should not be renormalized. From the inspection of the kernel function (5) two additional relations

$$
1=Z_{u_{1}} Z_{D}, \quad 1=Z_{u_{1}} Z_{g_{1}} Z_{D}^{3} Z_{v}^{-2}
$$

follow, which have to be satisfied to all orders in the perturbation scheme.

\section{FIXED POINTS AND SCALING REGIMES}

Once the renormalization procedure to a given order of perturbation scheme is performed, we can find the scaling behavior in the infrared IR limit by studying the flow as $\mu \rightarrow 0$. According to the general statement of the RG theory [37, 39, a possible IR asymptotic behavior is governed by the fixed point (FP) of the beta-functions.
All fixed points can be found from a requirement that all beta-functions of the model simultaneously vanish

$$
\beta_{g_{1}}\left(g^{*}\right)=\beta_{g_{2}}\left(g^{*}\right)=\beta_{u_{1}}\left(g^{*}\right)=\beta_{u_{2}}\left(g^{*}\right)=\beta_{a}\left(g^{*}\right)=0,
$$

where $g^{*}$ stands for an entire set of charges $\left\{g_{1}^{*}, g_{2}^{*}, u_{1}^{*}, u_{2}^{*}, a^{*}\right\}$. In what follows, the asterisk will always refer to coordinates of some fixed point. Whether the given FP could be realized in physical systems (IR stable) or not (IR unstable) is determined by eigenvalues of the matrix $\Omega=\left\{\Omega_{i j}\right\}$ with the elements

$$
\Omega_{i j}=\frac{\partial \beta_{i}}{\partial g_{j}},
$$

where $\beta_{i}$ is a full set of beta-functions and $g_{j}$ is the full set of charges $\left\{g_{1}, g_{2}, u_{1}, u_{2}, a\right\}$. For the IR stable FP the real parts of the eigenvalues of the matrix $\Omega$ have to be strictly positive. In general, these conditions determine a region of stability for the given $\mathrm{FP}$ in terms of $\varepsilon, \eta$ and $y$.

Furthermore, to obtain the RG equation, one can exploit a fact that the bare Green functions are independent of the momentum scale $\mu$ [37. Applying the differential operator $\mu \partial_{\mu}$ at the fixed bare quantities leads to the following equation for the renormalized Green function $G_{R}$

$$
\left\{\mathcal{D}_{\mathrm{RG}}+N_{\psi} \gamma_{\psi}+N_{\tilde{\psi}} \gamma_{\tilde{\psi}}+N_{\boldsymbol{v}} \gamma_{\boldsymbol{v}}\right\} G_{R}(e, \mu, \ldots)=0,
$$

where $G_{R}$ is a function of the full set $e$ of renormalized counterparts to the bare parameters $e_{0}=$ $\left\{D_{0}, \tau_{0}, u_{10}, u_{20}, g_{10}, g_{20}, a_{0}\right\}$, the reference mass scale $\mu$ and other parameters, e.g. spatial and time variables. The $R G$ operator $\mathcal{D}_{\mathrm{RG}}$ is given by

$$
\left.\mathcal{D}_{\mathrm{RG}} \equiv \mu \partial_{\mu}\right|_{0}=\mu \partial_{\mu}+\sum_{g} \beta_{g} \partial_{g}-\gamma_{D} \mathcal{D}_{D}-\gamma_{\tau} \mathcal{D}_{\tau},
$$

where $g \in\left\{g_{1}, g_{2}, u_{1}, u_{2}, a\right\}, \mathcal{D}_{x}=x \partial_{x}$ for any variable $x,\left.\ldots\right|_{0}$ stands for fixed bare parameters and $\gamma_{x}$ are the so-called anomalous dimensions of the quantity $x$ defined as

$$
\left.\gamma_{x} \equiv \mu \partial_{\mu} \ln Z_{x}\right|_{0}
$$

The beta-functions, which express the flows of parameters under the RG transformation [37, are defined through

$$
\beta_{g}=\left.\mu \partial_{\mu} g\right|_{0} .
$$

Applying this definition to relations (27) yields

$$
\begin{aligned}
\beta_{g_{1}} & =g_{1}\left(-y+2 \gamma_{D}-2 \gamma_{v}\right), & & \beta_{g_{2}}=g_{2}\left(-\varepsilon-\gamma_{g_{2}}\right), \\
\beta_{u_{1}} & =u_{1}\left(-\eta+\gamma_{D}\right), & & \beta_{u_{2}}=-u_{2} \gamma_{u_{2}}, \\
\beta_{a} & =-a \gamma_{a} . & &
\end{aligned}
$$

The last equation suggests that for the fixed points' equation $\beta_{a}\left(g^{*}\right)=0$ either $a=0$ or $a \neq 0$ has to be satisfied. 
However, as the explicit results (B4) show, this is not true (parameter $a$ appears also in the denominator of $\gamma_{a}$ ) and the right-hand side of $\beta_{a}$ has to be considered as a whole expression. A similar reasoning also applies for the function $\beta_{u_{2}}$.

It turns out that for some fixed points the computation of the eigenvalues of the matrix (33) is cumbersome and rather unpractical. In those cases it is possible to obtain information about the stability from analyzing RG flow equations [39. Its essential idea is to study a set of invariant charges $\bar{g}=\bar{g}(s, g)$ with the initial data $\left.\bar{g}\right|_{s=1}=g$. The parameter $s$ stands for a scaling parameter and one is interested in the behavior of charges in the limit $s \rightarrow 0$. The evolution of invariant charges is given by the equation

$$
\mathcal{D}_{s} \bar{g}=\beta(\bar{g}) .
$$

The very existence of IR stable solutions of the RG equations leads to the existence of the scaling behavior of Green functions. In dynamical models, critical dimensions of the quantity $Q$ is given by the relations

$$
\Delta_{Q}=d_{Q}^{k}+\Delta_{\omega} d_{Q}^{\omega}+\gamma_{Q}^{*}, \quad \Delta_{\omega}=2-\gamma_{D}^{*} .
$$

The $d_{k}^{Q}$ and $d_{\omega}^{Q}$ are canonical dimensions of the quantity $Q$ calculated with the help of Tab. II $\gamma_{Q}^{*}$ is the value of its anomalous dimension. Using Eqs. (40) we obtain the following relations

$$
\Delta_{\tilde{\psi}}=\frac{d}{2}+\gamma_{\tilde{\psi}}, \quad \Delta_{\psi}=\frac{d}{2}+\gamma_{\psi}, \quad \Delta_{\tau}=2+\gamma_{\tau}^{*} .
$$

Important information about the physical system can be read out from the behavior of correlation functions, which can be expressed in terms of the cumulant Green functions. In the percolation problems one is typically interested [2, 8] in the behavior of the following functions

a) The number $N(t, \tau)$ of active particles generated by a seed at the origin

$$
N(t)=\int d^{d} x G_{\psi \tilde{\psi}}(t, \boldsymbol{x}) .
$$

b) The mean square radius $R^{2}(t)$ of percolating particles, which started from the origin at time $t=0$

$$
R^{2}(t)=\frac{\int d^{d} \boldsymbol{x} \boldsymbol{x}^{2} G_{\psi \tilde{\psi}}(t, \boldsymbol{x})}{2 d \int d^{d} \boldsymbol{x} G_{\psi \tilde{\psi}}(t, \boldsymbol{x})} .
$$

c) Survival probability $P(t)$ of an active cluster originating from a seed at the origin

$$
P(t)=-\lim _{k \rightarrow \infty}\left\langle\tilde{\psi}(-t, \mathbf{0}) \mathrm{e}^{-k \int d^{d} \boldsymbol{x} \psi(0, \boldsymbol{x})}\right\rangle .
$$

By straightforward analysis [8] it can be shown that the scaling behavior of these functions is given by the asymptotic relations

$$
R^{2}(t) \sim t^{2 / \Delta_{\omega}}
$$

$$
\begin{aligned}
& N(t) \sim t^{-\left(\gamma_{\psi}+\gamma_{\tilde{\psi}}\right) / \Delta_{\omega}}, \\
& P(t) \sim t^{-\left(d+\gamma_{\psi}+\gamma_{\tilde{\psi}}\right) / 2 \Delta_{\omega}} .
\end{aligned}
$$

From the structure of anomalous dimensions (B4 B5) it is clear that the resulting system of equations for FPs is quite complicated. Although to some extent it is possible to obtain coordinates of the fixed points, the eigenvalues of the matrix (33) pose a more severe technical problem. Hence, in order to gain some physical insight into the structure of the model, we divide overall analysis into special cases and analyze them separately.

\section{A. Rapid change}

First, we perform an analysis of the rapid-change limit of the model. It is convenient [28, 36] to introduce the new variables $g_{1}^{\prime}$ and $w$ given by

$$
g_{1}^{\prime}=\frac{g_{1}}{u_{1}}, \quad w=\frac{1}{u_{1}} .
$$

The rapid change limit then corresponds to fixed points with a coordinate $w^{*}=0$. Using the definition (37) together with expressions (38) the beta-functions for the charges (48) are easily obtained

$$
\beta_{g_{1}^{\prime}}=g_{1}^{\prime}\left(\eta-y+\gamma_{D}-2 \gamma_{v}\right), \quad \beta_{w}=w\left(\eta-\gamma_{D}\right) .
$$

Analyzing the resulting system of equations seven possible regimes can be found. Their coordinates are listed in Tab. III in Appendix C. Due to the cumbersome form of the matrix (33), we were not able to determine all the corresponding eigenvalues in an explicit form. In particular, for nontrivial fixed points (with non-zero coordinates of $g_{1}^{\prime}, g_{2}$ and $u_{2}$ ) the resulting expressions are of a quite unpleasant form. Nevertheless, using numerical software [56] it is possible to obtain all the necessary information about the fixed points' structure and in this way the boundaries between the corresponding regimes have been obtained. In the analysis it is advantageous to exploit additional constraints following from the physical interpretation of the charges. For example, $g_{1}^{\prime}$ describes the density of kinetic energy of the velocity fluctuations, $g_{2}$ is equal to $\lambda^{2}$ and $a^{\prime}$ will be later on introduced (see Appendix C as $(1-2 a)^{2}$. Hence, it is clear that these parameters have to be non-negative real numbers. Fixed points that violate this condition can be immediately discarded as non-physical.

Out of seven possible fixed points, only four are IR stable: $\mathrm{FP}_{1}^{\mathrm{I}}, \mathrm{FP}_{2}^{\mathrm{I}}, \mathrm{FP}_{5}^{\mathrm{I}}$ and $\mathrm{FP}_{6}^{\mathrm{I}}$. Thus, only regimes which correspond to those points could be in principle realized in real physical systems. As expected [36, the coordinates of these fixed points (see Tab. III) and the scaling behavior of the Green functions (see Tab. VII) depend only on the parameter $\xi=y-\eta$. In what follows, we restrict our discussion only to them.

The $\mathrm{FP}_{1}^{\mathrm{I}}$ represents the free (Gaussian) FP for which all interactions are irrelevant and ordinary perturbation 


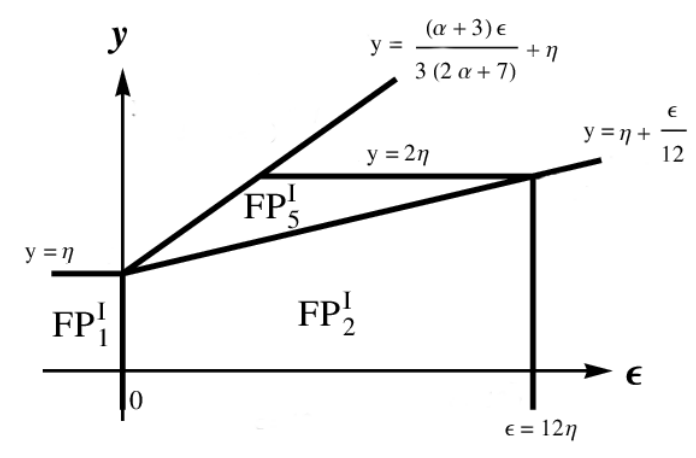

Figure 4. A qualitative sketch of the regions of stability for the fixed points in the limit of the rapid-change model. The borders between the regions are depicted with the bold lines.

theory is applicable. As expected, this regime is IR stable in the region

$$
y<\eta, \quad \eta>0, \quad \varepsilon<0 .
$$

The latter condition ensures that we are above the upper critical dimension $d_{c}=4$. For $\mathrm{FP}_{2}^{\mathrm{I}}$ the correlator of the velocity field is irrelevant and this point describes standard the DP universality class [8] and is IR stable in the region

$$
\varepsilon>0, \quad \varepsilon / 12+\eta>y, \quad \varepsilon<12 \eta .
$$

The remaining two fixed points constitute nontrivial regimes for which velocity fluctuations as well as percolation interaction become relevant. The $\mathrm{FP}_{5}^{\mathrm{I}}$ is IR stable in the region given by

$$
(\alpha+3) \varepsilon>3(2 \alpha+7)(y-\eta), \quad 12(y-\eta)>\varepsilon, \quad 2 \eta>y .
$$

The boundaries for $\mathrm{FP}_{6}^{\mathrm{I}}$ can be only computed by numerical calculations.

Using the information about the phase boundaries, a qualitative picture of the phase diagram can be constructed. In Fig. 4 the situation in the plane $(\varepsilon, y)$ is depicted. We observe that compressibility affects only the outer boundary of $\mathrm{FP}_{5}^{\mathrm{I}}$. The larger value of $\alpha$ the larger area of stability. We also observe that the realizability of the regime $\mathrm{FP}_{5}^{\mathrm{I}}$ crucially depends on the nonzero value of $\eta$.

The important subclass of the rapid-change limit constitutes thermal velocity fluctuations, which are characterized by the quadratic dispersion law [57. In our formulation this is achieved by considering the following relation:

$$
\eta=6+y-\varepsilon
$$

which follows directly from expression (7). The situation for increasing values of the parameter $\alpha$ is depicted in Fig. 5. We see that for physical space dimensions $d=3(\varepsilon=1)$ and $d=2(\varepsilon=2)$ the only stable regime
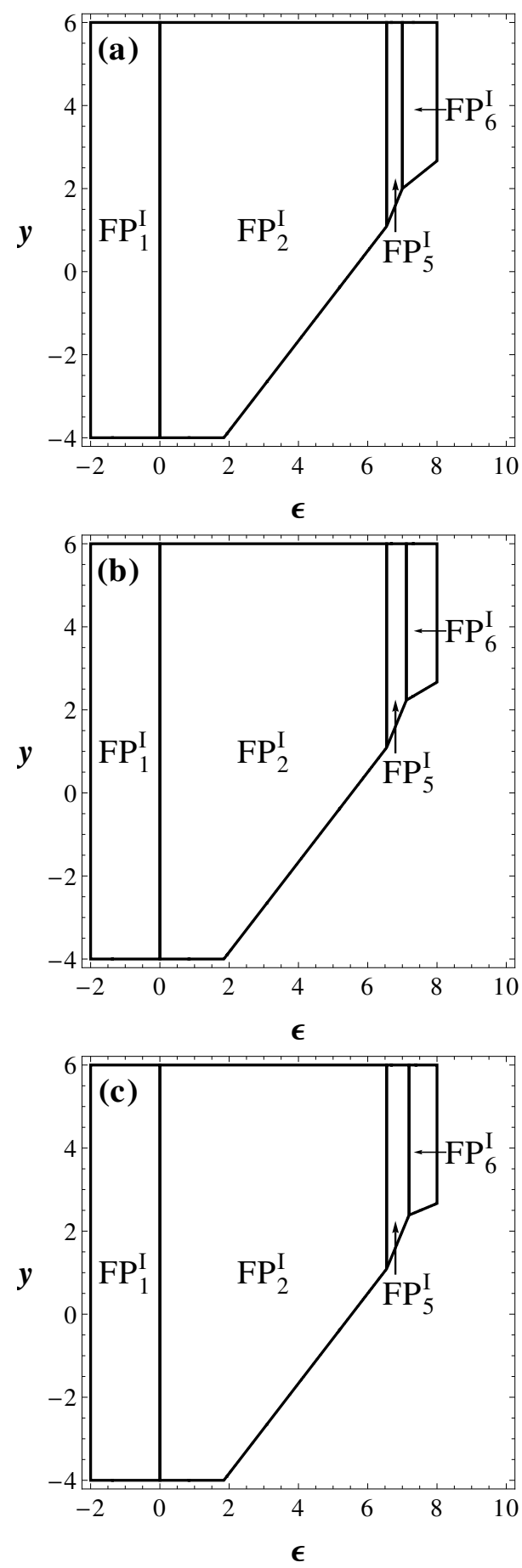

Figure 5. Fixed points' structure for the thermal noise situation (53). From above to bottom the compressibility parameter $\alpha$ attains consecutively the values: (a) $\alpha=0$, (b) $\alpha=5$ and (c) $\alpha=100$.

is that of pure DP. The nontrivial regimes $\mathrm{FP}_{5}^{\mathrm{I}}$ and $\mathrm{FP}_{6}^{\mathrm{I}}$ are realized only in the nonphysical region for large values of $\varepsilon$. This numerical result confirms our previous expectations [40, 41]. It was pointed out [58, 59] that genuine thermal fluctuations could change IR stability of 
the given universality class. However, this is not realized for the percolation process.

\section{B. Regime of frozen velocity field}

According to equation (8), the regime of the frozen velocity field corresponds to the constraint $u_{1}^{*}=0$. Using

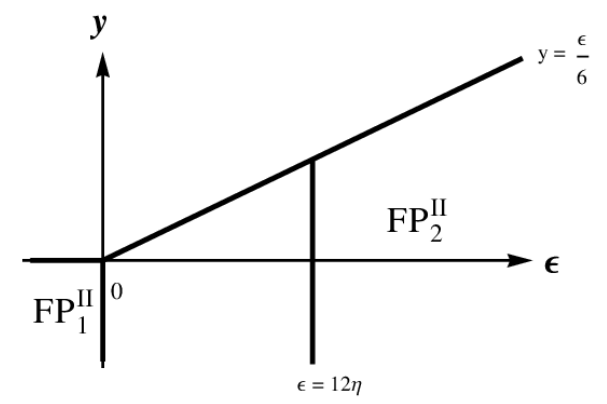

Figure 6. Regions of stability for the fixed points in the limit of the frozen velocity field. The borders between the regions are depicted with the bold lines.

a general form of anomalous dimensions (B4) with the given constraint on $u_{1}$ eight possible fixed points are obtained. Their coordinates are listed in Tab. [IV] in Appendix $\mathrm{C}$. However, only three of them $\left(\mathrm{FP}_{1}^{\mathrm{II}}, \mathrm{FP}_{2}^{\mathrm{II}}\right.$ and $\mathrm{FP}_{7}^{\mathrm{II}}$ ) could be physically realized (IR stable).

The fixed point $\mathrm{FP}_{1}^{\mathrm{II}}$ describes the free (Gaussian) theory. It is stable in the region

$$
y<0, \quad \varepsilon<0, \quad \eta<0
$$

For $\mathrm{FP}_{2}^{\mathrm{II}}$ the velocity field is asymptotically irrelevant and the only relevant interaction is due to the percolation process itself. This regime is stable in the region

$$
\varepsilon>6 y, \quad \varepsilon>0, \quad \varepsilon>12 \eta .
$$

On the other hand, $\mathrm{FP}_{7}^{\mathrm{II}}$ represents a truly nontrivial regime for which both velocity and percolation are relevant. The regions of stability for the $\mathrm{FP}_{1}^{\mathrm{II}}$ and $\mathrm{FP}_{2}^{\mathrm{II}}$ are depicted in Fig. 6. Since for these two points the velocity field could be effectively neglected, the trivial observation is that these boundaries do not depend on the value of the parameter $\alpha$. The stability region of $\mathrm{FP}_{7}^{\mathrm{II}}$ can be computed only numerically.

In order to study the influence of compressibility on the stability in the nontrivial regime $\mathrm{FP}_{7}^{\mathrm{II}}$, we have studied situation for $\eta=0$. For other values of $\eta$ the situation remains qualitatively the same. The situation for increasing values of $\alpha$ is depicted in Fig. 7. We observe that for $\alpha=0$ there is a region of stability for $\mathrm{FP}_{7}^{\mathrm{II}}$, which shrinks for the immediate value $\alpha=3.5$ to a smaller area. $\mathrm{Nu}-$ merical analysis shows that this shrinking continues well down to the value $\alpha=6$. A further increase of $\alpha$ leads to a substantially larger region of stability for the given FP. Already for $\alpha=8$ this region covers all the rest of
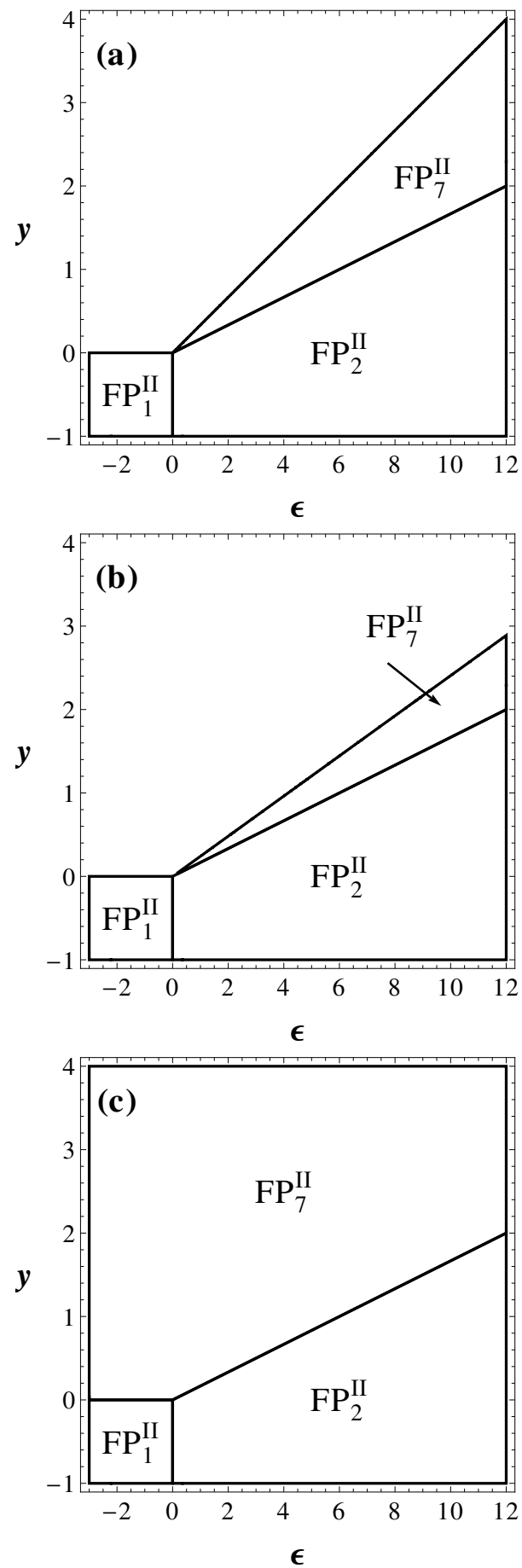

Figure 7. Fixed points' structure for frozen velocity case with $\eta=0$. From above to bottom the compressibility parameter $\alpha$ attains consecutively the values: (a) $\alpha=0,-$ (b) $\alpha=3.5$ and (c) $\alpha=8$.

the $(y, \varepsilon)$ plane. The compressibility thus changes profoundly a simple picture expected from an incompressible case. Altogether the advection process becomes more efficient due to the combined effects of compressibility and the nonlinear terms. 


\section{Turbulent advection}

In the last part we focus on a special case of the turbulent advection. Our main aim is to determine whether Kolmogorov regime [25], which corresponds to the choice $y=2 \eta=8 / 3$, could lead to a new nontrivial regime for the percolation process. In this section, the parameter $\eta$ is always considered to attain its Kolmogorov value, 4/3. For a better visualization we present two-dimensional regions of stability in the plane $(\varepsilon, y)$ for different values of the parameter $\alpha$.

First of all, we reanalyze the situation for the rapidchange model. The result is depicted in Fig. 8. It is clearly visible that for this case a realistic turbulent scenario $(\varepsilon=1$ or $\varepsilon=2$ ) falls out of the possible stable regions. This result is expected because the rapid-change model with vanishing time-correlations could not properly describe well-known turbulent properties [25, 26]. We also observe that compressibility mainly affects the boundaries between the regions $\mathrm{FP}_{5}^{\mathrm{I}}$ and $\mathrm{FP}_{6}^{\mathrm{I}}$. However, this happens mainly in the nonphysical region.

Next, we turn our attention to a similar analysis for the frozen velocity field. The corresponding stability regions are depicted in Fig. 9. Here we see that the situation is more complex. The regime $\mathrm{FP}_{2}^{\mathrm{II}}$ is situated in the non-physical region and could not be realized. For small values of the parameter $\alpha$ the Kolmogorov regime (depicted by a point) does not belong to the frozen velocity limit. However, from a special value $\alpha=6$ up to $\alpha \rightarrow \infty$ the Kolmogorov regime belongs to the frozen velocity limit. Note that the bottom line for the region of stability of $\mathrm{FP}_{7}^{\mathrm{II}}$ is exactly given by $y=4 / 3$. We observe that compressibility affects mainly the boundary of the nontrivial region. We conclude that the presence of compressibility has a stabilizing effect on the regimes where nonlinearities are relevant.

Finally, we look carefully at the nontrivial regime, which means that no special requirements were laid upon the parameter $u_{1}$. As obtaining of analytical results proves to be too difficult, we have analyzed numerically the differential equations for the RG flow $(39)$. We found that the behavior of the RG flows is as follows. There exists a borderline in the plane $\left(\varepsilon, \alpha_{c}\right)$ given approximately by the expression

$$
\alpha_{c}=-12.131 \varepsilon+117.165 .
$$

Below $\alpha_{c}$, only the frozen velocity regime corresponding to $\mathrm{FP}_{7}^{\mathrm{II}}$ is stable. Above $\alpha_{c}$, three fixed points $\mathrm{FP}_{7}^{\mathrm{II}}$, $\mathrm{FP}_{1}^{\mathrm{III}}$ and $\mathrm{FP}_{2}^{\mathrm{III}}$ are observed. Whereas two of them $\left(\mathrm{FP}_{7}^{\mathrm{II}}\right.$ and $\mathrm{FP}_{1}^{\mathrm{III}}$ ) are IR stable, the remaining one $\mathrm{FP}_{2}^{\mathrm{III}}$ is unstable in the IR regime. Again one of the stable FPS corresponds to $\mathrm{FP}_{7}^{\mathrm{II}}$, but the new $\mathrm{FP}$ is a regime with finite correlation time. For the reference the coordinates of these two points for the value $\alpha=110$ are given in Tabs. V]and VI in Appendix C. Since all free parameters $(\varepsilon, \eta, y, \alpha)$ are the same for both points, which of the two points will be realized depends on the initial values of the bare parameters. A similar situation is observed for the
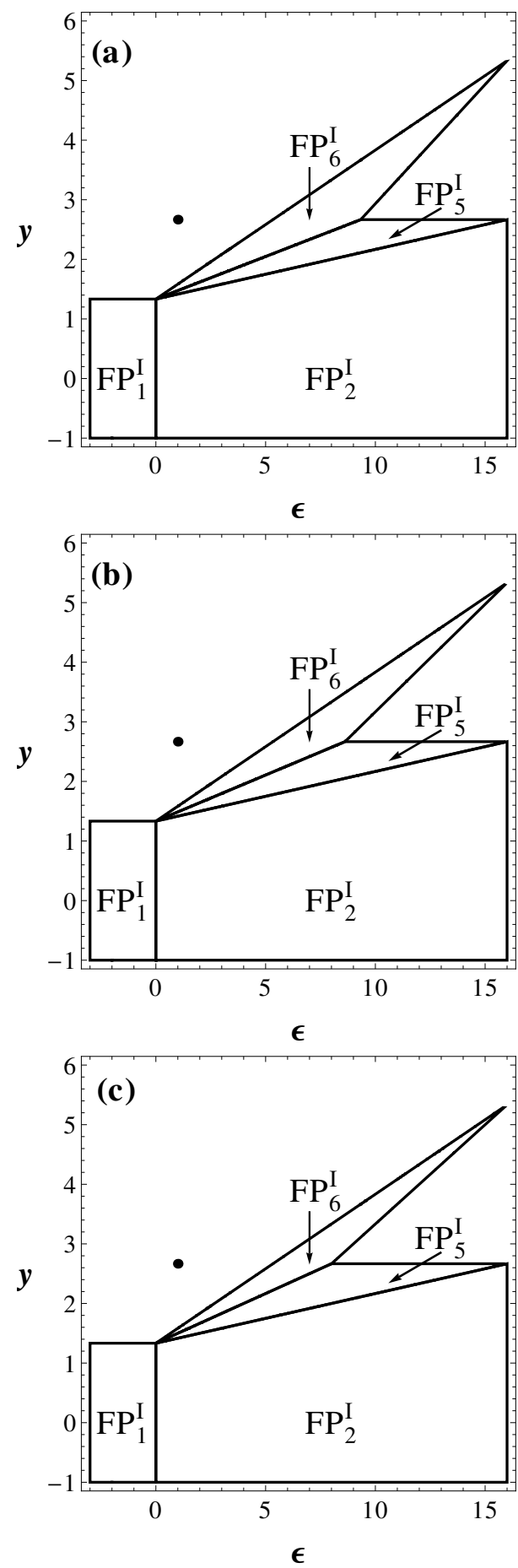

Figure 8. Fixed points' structure for rapid change model with $\eta=4 / 3$. From above to bottom the compressibility parameter $\alpha$ attains consecutively the values: (a) $\alpha=0$, (b) $\alpha=5$ and (c) $\alpha=\infty$. The dot denotes the coordinates of the three-dimensional Kolmogorov regime.

stochastic magnetohydrodynamic turbulence 60, where the crucial role is played by a forcing decay-parameter.

For illustration purposes the projections of the RG flow onto the planes $\left(g_{1}, u_{1}\right)$ and $\left(g_{2}, u_{1}\right)$ are depicted in Fig. 

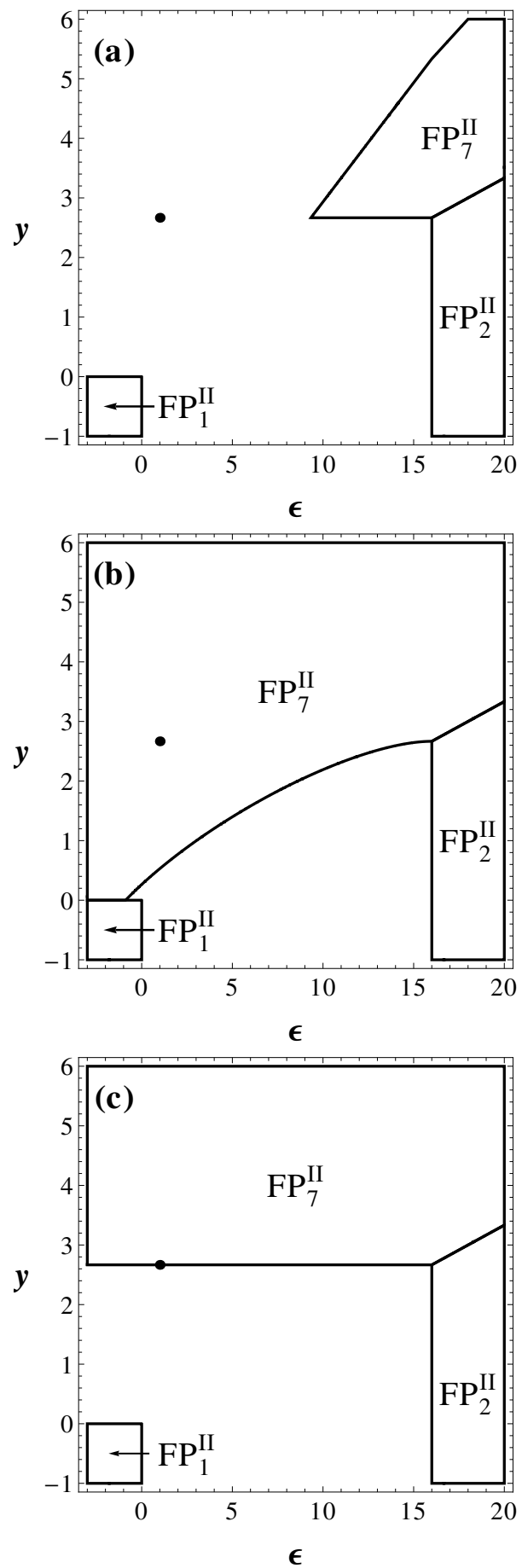

Figure 9. Fixed points' structure for the frozen velocity case with $\eta=4 / 3$. From above to bottom the compressibility parameter $\alpha$ attains consecutively the values: (a) $\alpha=0$, (b) $\alpha=8$ and (c) $\alpha=\infty$. The dot denotes the coordinates of the three-dimensional Kolmogorov regime.

10. The two stable points are clearly separated by the unstable one.
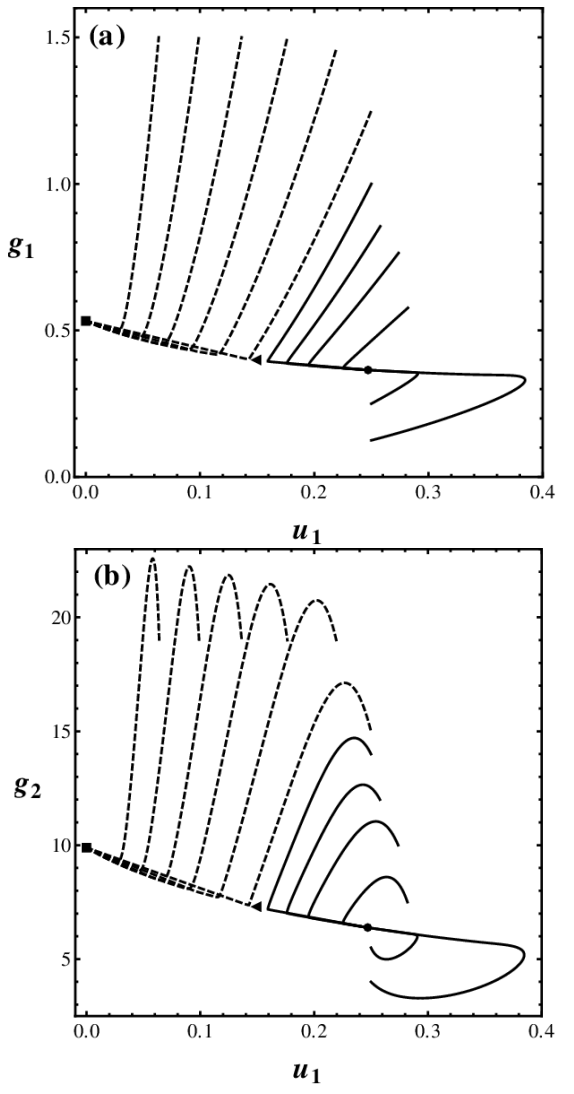

Figure 10. Demonstration of the RG trajectories flows' in: (a) the plane $\left(g_{1}, u_{1}\right)$ and (b) the plane $\left(g_{2}, u_{1}\right)$ for three dimensional $(\varepsilon=1)$ turbulent advection with $\alpha=110$. The square denotes frozen velocity regime $\mathrm{FP}_{7}^{\mathrm{II}}$, triangle 4 corresponds to the unstable regime $\mathrm{FP}_{2}^{\mathrm{III}}$ and circle $\bullet$ to the nontrivial regime $\mathrm{FP}_{1}^{\mathrm{III}}$ for which the time correlations are relevant. Dashed lines corresponds to the chosen flows to the point $\mathrm{FP}_{7}^{\mathrm{II}}$, whereas the full lines to the flows to the other stable point $\mathrm{FP}_{1}^{\mathrm{III}}$.

\section{CONCLUSIONS}

In this paper, we have studied an effect of compressibility on the paradigmatic model of percolation spreading. The coarse grained model of percolation with inclusion of the advecting velocity field can be reformulated as the multiplicatively renormalizable field theoretic model.

We have found that depending on the values of a spatial dimension $d=4-\varepsilon$, scaling exponents $y$ and $\eta$, describing statistics of velocity fluctuations and a degree of compressibility $\alpha$, the model exhibits 8 distinct universality classes. Some of them are already well-known: the Gaussian (free) fixed point, a directed percolation without advection and a passive scalar advection. The remaining points correspond to new universality classes, for which an interplay between advection and percolation is relevant.

It also has to be kept in mind that only relatively small values of $\alpha$ are allowed $(\alpha \ll 1)$ in our model. It corresponds to small fluctuations of the density $\rho$, what is 
tacitly supposed in our investigation. In other words, it is assumed that the stochastic component of the velocity field of the fluid is much smaller than the velocity of the sound in the system (the Mach number Ma $\ll 1$ ). Hence our results must be taken with a grain of salt. Nevertheless we believe that a qualitative picture for large values of compressibility should remain the same. In order to properly describe effects of strong compressibility and to better understand non-universal effects for turbulent advection one should proceed one step further and employ a more sophisticated model for velocity fluctuations, e.g. one introduced in the works [61, 62 and later used for the passive advection problem in [63]. However, such a model goes well beyond the aim of this paper and its detailed study is left for a future study.

\section{Acknowledgments}

The work was supported by VEGA grant No. $1 / 0222 / 13$ of the Ministry of Education, Science, Research and Sport of the Slovak Republic. N.V.A. and A.S.K. acknowledge Saint Peterburg State University for Research Grant No. 11.38.185.2014. A.S.K. was also supported by the grant 16-32-00086 provided by the Russian Foundation for Basic Research.

\section{Appendix A: Calculation of the Renormalization Constants}

In this appendix, we describe in detail how the renormalization constants are computed.

Though the bare action (9) contains a lot of terms, the number of divergent Feynman graphs is quite low to the first order of perturbation theory. Their analysis is to some extent simplified by two facts:

1. Integral of a power of internal momenta is zero in the dimensional regularization. Hence, the tadpole diagrams are discarded.

2. Closed circuits of propagators $\tilde{\psi} \psi$ vanish identically, which is a consequence of the Itô time discretization 64, 65, which we consider here.

For the two-point Green functions $\Gamma_{\tilde{\psi} \psi}$, the following Dyson equation can be written

$$
\Gamma_{\tilde{\psi} \psi}=i \omega Z_{1}-D p^{2} Z_{2}-D \tau Z_{3}+\overbrace{1}^{1}
$$

Up to the one-loop order the perturbation expansions for the vertex functions read consequently

$$
\Gamma_{\tilde{\psi} \psi \boldsymbol{v}}=-i p_{j} Z_{4}-i a q_{j} Z_{5}+\cdots
$$
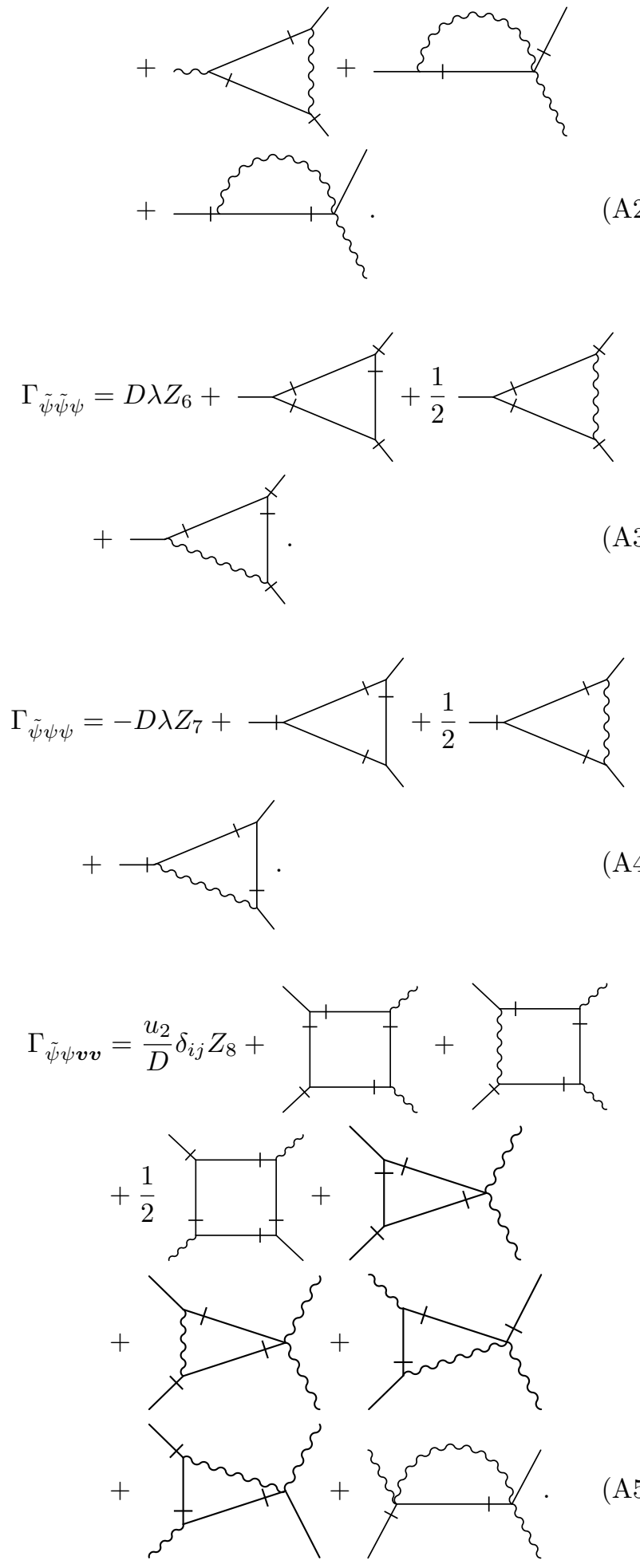

In these equations, we have explicitly given the symmetry coefficients 39] of the corresponding diagrams. The numerical contributions arising from variational derivatives with respect to the external fields are included in the contribution of a given graph. Note that in the language of Feynman graphs the need for the term $\propto \tilde{\psi} \psi \boldsymbol{v}^{2}$ can be traced out to the presence of the second Feynman graph in (A5), which does not vanish due to finite correlation time property of the velocity propagator (4). 
The computation of the diverging parts of the Feynman graphs follows the standard methods of dimensional regularization [37, 39] and the 1-loop results are

$$
\begin{array}{rl}
Z_{1} & =1+\frac{g_{1} \alpha a(1-a)}{\left(1+u_{1}\right)^{2} y}+\frac{g_{2}}{4 \varepsilon}, \\
Z_{2} & =1-\frac{g_{1}}{4\left(1+u_{1}\right) y}\left[3+\alpha\left(\frac{u_{1}-1}{u_{1}+1}-\frac{4 a(1-a) u_{1}}{\left(1+u_{1}\right)^{2}}\right)\right] \\
& +\frac{g_{2}}{8 \varepsilon}, \\
Z_{3} & =1+\frac{g_{1} \alpha a(1-a)}{\left(1+u_{1}\right)^{2} y}+\frac{g_{2}}{2 \varepsilon}, \\
Z_{4} & =1+\frac{g_{1}}{4\left(1+u_{1}\right)^{2} y}\left[\alpha\left(1+\frac{4 a(1-a) u_{1}}{1+u_{1}}\right)\right. \\
& \left.-u_{2}\left(6+6 u_{1}+2 \alpha u_{1}\right)\right]+\frac{g_{2}}{4 \varepsilon}, \\
Z_{5} & =1+\frac{g_{1} \alpha}{4\left(1+u_{1}\right)^{2} y}\left[1+2(1-a)\left(2 a-\frac{1}{1+u_{1}}\right)\right] \\
& -\frac{g_{1} u_{2}}{4 a\left(1+u_{1}\right) y}\left[3+\alpha-\frac{2 \alpha(1-a)}{1+u_{1}}\right]+\frac{g_{2}(4 a-1)}{8 a \varepsilon} \\
Z_{6} & =1-\frac{g_{1} \alpha(1-a)}{\left(1+u_{1}\right) y}\left[1-a-\frac{2 a}{1+u_{1}}\right]+\frac{g_{2}}{\varepsilon}, \\
Z_{7} & =1-\frac{g_{1} \alpha a}{\left(1+u_{1}\right) y}\left[a-\frac{2(1-a)}{1+u_{1}}\right]+\frac{g_{2}}{\varepsilon}, \\
2\left(1+u_{1}\right) y & 2 a(1-a)+1 \\
\left.Z_{2}(3+\alpha)\right]+\frac{g_{2}}{2 \varepsilon} . & \alpha a(1-a) \\
u_{2}\left(1+u_{1}\right)^{2}
\end{array}
$$

The ubiquitous geometric factor stemming from the angular integration is included into the renormalized charges $g_{1}$ and $g_{2}$ via the following redefinitions

$$
\frac{g_{1} S_{d}}{2(2 \pi)^{d}} \rightarrow g_{1}, \quad \frac{g_{2} S_{d}}{2(2 \pi)^{d}} \rightarrow g_{2}
$$

where

$$
S_{d}=\frac{2 \pi^{d / 2}}{\Gamma(d / 2)}
$$

is the surface area of the unit sphere in the $d$-dimensional space and $\Gamma(x)$ is Euler's Gamma function. Equations A6 have to satisfy certain conditions dictated by the symmetry of the model given by (24) and (26). This symmetry results into the following conditions 40 for the renormalization constants:

$$
\begin{aligned}
& Z_{i}(a)=Z_{i}(1-a), \quad i \in\{1,2,3,4,8\} \\
& Z_{6}(a)=Z_{7}(1-a), \\
& Z_{7}(a)=Z_{6}(1-a), \\
& Z_{1}(a)-a Z_{5}(a)=(1-a) Z_{5}(1-a),
\end{aligned}
$$

where the RG constants are considered as functions of the renormalized parameter $a$. By direct inspection of (A6), it is easy to see that they indeed fulfill these requirements.
Further, relations 30 could be inverted with respect to the RG constants for the fields and parameters in a straightforward manner to yield

$$
\begin{array}{rlrl}
Z_{D} & =Z_{2} Z_{1}^{-1}, & Z_{\tau} & =Z_{3} Z_{2}^{-1}, \\
Z_{v} & =Z_{4} Z_{1}^{-1}, & Z_{a} & =Z_{5} Z_{4}^{-1}, \\
Z_{\psi} & =Z_{1}^{1 / 2} Z_{6}^{-1 / 2} Z_{7}^{1 / 2}, & Z_{\tilde{\psi}} & =Z_{1}^{1 / 2} Z_{6}^{1 / 2} Z_{7}^{-1 / 2}, \\
Z_{u_{1}} & =Z_{1} Z_{2}^{-1}, & Z_{\lambda} & =Z_{1}^{-1 / 2} Z_{2}^{-1} Z_{6}^{1 / 2} Z_{7}^{1 / 2}, \\
Z_{g_{2}} & =Z_{1}^{-1} Z_{2}^{-2} Z_{6} Z_{7}, & Z_{u_{2}} & =Z_{2} Z_{8} Z_{4}^{-2}, \\
Z_{g_{1}} & =Z_{2}^{-2} Z_{4}^{2} . &
\end{array}
$$

After insertion of explicit results for renormalization constants A6, one obtains the desired RG constants of the fields and parameters of the model.

\section{Appendix B: Anomalous dimensions}

In this section, we review the explicit expressions for the anomalous dimension $\gamma_{x}, x \in\left\{g_{1}, g_{2}, u_{1}, u_{2}, a\right\}$ of the charges and for the fields $x \in\{\psi, \tilde{\psi}, \boldsymbol{v}\}$, respectively. From relations (A10), the following expressions directly follow:

$$
\begin{aligned}
\gamma_{D} & =-\gamma_{1}+\gamma_{2}, & \gamma_{a} & =-\gamma_{4}+\gamma_{5}, \\
\gamma_{u_{2}} & =\gamma_{2}-2 \gamma_{4}+\gamma_{8}, & \gamma_{\boldsymbol{v}} & =-\gamma_{1}+\gamma_{4}, \\
\gamma_{g_{2}} & =-\gamma_{1}-2 \gamma_{2}+\gamma_{6}+\gamma_{7}, & \gamma_{g_{1}} & =2 \gamma_{4}-2 \gamma_{2}, \\
\gamma_{\tau} & =\gamma_{3}-\gamma_{2} . & &
\end{aligned}
$$

The anomalous dimension $\gamma_{x}$, corresponding to the renormalization constant $Z_{x}, x \in\{1,2, \ldots, 8\}$ can be found from the approximate relation

$$
\begin{aligned}
\gamma_{x} & =\left.\mu \partial_{\mu} \ln Z_{x}\right|_{0}=\left(\beta_{g_{1}} \partial_{g_{1}}+\beta_{g_{2}} \partial_{g_{2}}\right) \ln Z_{x} \\
& \approx-\left(y g_{1} \partial_{g_{1}}+\varepsilon g_{2} \partial_{g_{2}}\right) \ln Z_{i} .
\end{aligned}
$$

We have subsequently taken into account the following facts: definitions (36) and (37), $Z_{i}$ could depend only on dimensionless coupling constants and we have retained only the leading order terms in $\beta$-functions, which is sufficient in the one-loop approximation. Note that $-\eta \mathcal{D}_{u_{1}}$ has not been included due to the absence of a pole in $\eta$. As discussed in literature [28, this is a property of the low-order perturbation theory.

\section{General case}

The anomalous dimensions for the charges of theory read

$$
\begin{aligned}
\gamma_{g_{1}} & =-\frac{g_{1}}{2\left(1+u_{1}\right)^{2}}\left[3\left(1+u_{1}\right)-u_{2}\left(6+6 u_{1}+2 \alpha u_{1}\right)\right. \\
& \left.+\alpha u_{1}\right]-\frac{g_{2}}{4},
\end{aligned}
$$




$$
\begin{aligned}
\gamma_{D} & =\frac{g_{1}}{4\left(1+u_{1}\right)}\left[3+\alpha \frac{u_{1}-1}{u_{1}+1}+\frac{4 \alpha a(1-a)}{\left(1+u_{1}\right)^{2}}\right]+\frac{g_{2}}{8}, \\
\gamma_{a} & =(1-2 a)\left[\frac{g_{1} \alpha(1-a)}{2\left(1+u_{1}\right)^{3}}+\frac{g_{1} u_{2}}{4 a\left(1+u_{1}\right)}(3+\alpha\right. \\
& \left.\left.-\frac{2 \alpha}{1+u_{1}}\right)+\frac{g_{2}}{8 a}\right], \\
\gamma_{u_{2}} & =\frac{g_{1}\left(1-2 u_{2}\right)}{4\left(1+u_{1}\right)}\left[3+\alpha \frac{u_{1}-1}{u_{1}+1}+\frac{2 \alpha a(1-a)}{u_{2}\left(1+u_{1}\right)^{2}}\right]-\frac{g_{2}}{8}, \\
\gamma_{g_{2}} & =-\frac{3 g_{1}}{2\left(1+u_{1}\right)}+\frac{g_{1} \alpha}{1+u_{1}}\left[\frac{(1-2 a)^{2}}{2}+\frac{1-3 a(1-a)}{1+u_{1}}\right. \\
& \left.+\frac{2 a(1-a) u_{1}}{\left(1+u_{1}\right)^{2}}\right]-\frac{3 g_{2}}{2}, \\
\gamma_{\tau} & =-\frac{g_{1}}{4\left(1+u_{1}\right)}\left[3+\frac{\alpha}{u_{1}+1}\left(u_{1}-1+\frac{4 a(1-a)}{1+u_{1}}\right)\right] \\
& -\frac{3 g_{2}}{8} \cdot
\end{aligned}
$$

In a similar manner anomalous dimensions for the fields can be computed. The resulting expressions then read

$$
\begin{aligned}
\gamma_{\psi} & =\frac{g_{1} \alpha}{2\left(1+u_{1}\right)^{2}}\left[-a(1-a)+\left(1+u_{1}\right)(2 a-1)\right]-\frac{g_{2}}{8} \\
\gamma_{\tilde{\psi}} & =\frac{g_{1} \alpha}{2\left(1+u_{1}\right)^{2}}\left[-a(1-a)+\left(1+u_{1}\right)(1-2 a)\right]-\frac{g_{2}}{8} \\
\gamma_{\boldsymbol{v}} & =\frac{g_{1} \alpha}{4\left(1+u_{1}\right)^{2}}\left[\frac{4 a(1-a)}{1+u_{1}}-1\right]+\frac{g_{1} u_{2}}{2\left(1+u_{1}\right)} \\
& \times\left[3+\frac{\alpha u_{1}}{1+u_{1}}\right] .
\end{aligned}
$$

\section{Rapid-change model}

Introducing new variables through (48) in relations (B4), following relations for anomalous dimensions

$$
\begin{aligned}
\gamma_{g_{1}} & =-\frac{g_{1}^{\prime}}{2(1+w)^{2}}\left[3(1+w)-u_{2}(6 w+6+2 \alpha)+\alpha\right] \\
& -\frac{g_{2}}{4}, \\
\gamma_{D} & =\frac{g_{1}^{\prime}}{4(1+w)}\left(3+\alpha \frac{1-w}{1+w}+\frac{4 \alpha a(1-a) w^{2}}{(1+w)^{2}}\right)+\frac{g_{2}}{8}, \\
\gamma_{a} & =(1-2 a)\left(\frac{g_{1}^{\prime} \alpha(1-a) w^{2}}{2(1+w)^{3}}+\frac{g_{1}^{\prime} u_{2}}{4 a(1+w)}[3+\alpha\right. \\
& \left.\left.-\frac{2 \alpha w}{1+w}\right]+\frac{g_{2}}{8 a}\right), \\
\gamma_{u_{2}} & =\frac{g_{1}^{\prime}\left(1-2 u_{2}\right)}{4(1+w)}\left(3+\alpha \frac{1-w}{1+w}+\frac{2 \alpha a(1-a) w^{2}}{u_{2}(1+w)^{2}}\right)-\frac{g_{2}}{8}, \\
\gamma_{g_{2}} & =-\frac{3 g_{1}^{\prime}}{2(1+w)}+\frac{g_{1}^{\prime} \alpha}{1+w}\left(\frac{(1-2 a)^{2}}{2}+w \frac{1-3 a(1-a)}{1+w}\right. \\
& \left.+\frac{2 a(1-a) w}{(1+w)^{2}}\right)-\frac{3 g_{2}}{2},
\end{aligned}
$$

$$
\begin{aligned}
\gamma_{\tau} & =\frac{g_{1}^{\prime}}{4(1+w)}\left[3+\frac{\alpha}{1+w}\left(1-w-\frac{4 a(1-a)}{1+w}\right.\right. \\
& \left.\times(w+2) w)^{2}\right]-\frac{5 g_{2}}{8} .
\end{aligned}
$$

are obtained. Anomalous dimensions for the fields are given by the expressions

$$
\begin{aligned}
\gamma_{\psi} & =\frac{g_{1}^{\prime} \alpha}{2(1+w)^{2}}[-a(1-a) w+(1+w)(2 a-1)]-\frac{g_{2}}{8} \\
\gamma_{\tilde{\psi}} & =\frac{g_{1}^{\prime} \alpha}{2(1+w)^{2}}[-a(1-a) w+(1+w)(1-2 a)]-\frac{g_{2}}{8} \\
\gamma_{\boldsymbol{v}} & =\frac{g_{1}^{\prime} \alpha w}{4(1+w)^{2}}\left(\frac{4 a(1-a) w}{1+w}-1\right)+\frac{g_{1}^{\prime} u_{2}}{2(1+w)} \\
& \times\left(3+\frac{\alpha}{1+w}\right) .
\end{aligned}
$$

\section{Appendix C: Coordinates of the fixed points}

In this section, we explicitly list analytical expressions for the coordinates of the fixed points. For convenience we have introduced a new parameter $a^{\prime}$ via the relation $a^{\prime}=(1-2 a)^{2}$. Here NF is an abbreviation for Not Fixed, i.e., for the given $\mathrm{FP}$ the corresponding value of a charge coordinate could not be unambiguously determined. In that case, the given FP rather corresponds to the whole line of FPs.

The fixed point $\mathrm{FP}_{6}^{\mathrm{II}}$ corresponds actually to the line of possible fixed points determined by the following system of equations:

$$
g_{1}^{*}\left(1-2 u_{2}^{*}\right)=\frac{2 y}{3}, \quad g_{1}^{*}\left(\alpha a^{\prime *}-3\right)=\frac{2 y}{3}(\alpha-3) .
$$

Further, the coordinates of the last two fixed points $\mathrm{FP}_{7}^{\mathrm{II}}$ and $\mathrm{FP}_{8}^{\mathrm{II}}$ are given by the following expressions:

$$
\begin{aligned}
g_{1}^{*} & =\frac{-4}{(\alpha-6)[(\alpha-12) \alpha-180]}\left[\left(\alpha^{2}-12 \alpha-72\right) \varepsilon\right. \\
& \left.+3\left(21 \alpha-2 \alpha^{2}+54\right) y \pm 9 A\right] \\
g_{2}^{*} & =-\frac{2\left[\left(21 \alpha-2 \alpha^{2}+54\right) y+36 \varepsilon \pm 3 A\right]}{(\alpha-12) \alpha-180}, \\
u_{2}^{*} & =\frac{4(\alpha-3) \varepsilon+(42-25 \alpha) y \pm A}{8(\alpha-6) \varepsilon-48(\alpha-3) y},
\end{aligned}
$$

where $A$ stands for the expression

$$
\begin{aligned}
A & =\left[-8\left(\alpha^{2}-9 \alpha+126\right) \varepsilon y+\left(49 \alpha^{2}-372 \alpha+1764\right) y^{2}\right. \\
& \left.+144 \varepsilon^{2}\right]^{1 / 2} .
\end{aligned}
$$

The plus sign in $\sqrt{\mathrm{C} 2}$ refers to the point $\mathrm{FP}_{7}^{\mathrm{II}}$, whereas the minus sign for $\mathrm{FP}_{8}^{\mathrm{II}}$. 


\begin{tabular}{|l|c|c|c|c|}
\hline $\mathrm{FP}^{\mathrm{I}}$ & $g_{1}^{\prime *}$ & $g_{2}^{*}$ & $u_{2}^{*}$ & $a^{\prime *}$ \\
\hline $\mathrm{FP}_{1}^{\mathrm{I}}$ & 0 & 0 & $\mathrm{NF}$ & $\mathrm{NF}$ \\
\hline $\mathrm{FP}_{2}^{\mathrm{I}}$ & 0 & $\frac{2 \varepsilon}{3}$ & 0 & 0 \\
\hline $\mathrm{FP}_{3}^{\mathrm{I}}$ & $\frac{4 \xi}{3+\alpha}$ & 0 & 0 & $\mathrm{NF}$ \\
\hline $\mathrm{FP}_{4}^{\mathrm{I}}$ & $-\frac{4 \xi}{3+\alpha}$ & 0 & $\frac{1}{2}$ & 0 \\
\hline $\mathrm{FP}_{5}^{\mathrm{I}}$ & $\frac{24 \xi-2 \varepsilon}{3(5+2 \alpha)}$ & $\frac{4 \varepsilon(3+\alpha)-24 \xi}{3(5+2 \alpha)}$ & 0 & 0 \\
\hline $\mathrm{FP}_{6}^{\mathrm{I}}$ & $\frac{2[\varepsilon-4 \xi]}{9+2 \alpha}$ & $\frac{4 \varepsilon(3+\alpha)+24 \xi}{3(9+2 \alpha)}$ & $\frac{(3+\alpha) \varepsilon-3 \xi(7+2 \alpha)}{3(3+\alpha)[\varepsilon-4 \xi]}$ & 0 \\
\hline $\mathrm{FP}_{7}^{\mathrm{I}}$ & $-\frac{\xi}{3+\alpha}$ & $2 \xi$ & 1 & $-\frac{3(5+2 \alpha)}{\alpha}+\frac{2(3+\alpha) \varepsilon}{\alpha \xi}$ \\
\hline
\end{tabular}

Table III. List of all fixed points obtained in the rapid-change limit. The coordinate $w^{*}$ is equal to 0 for all points.

\begin{tabular}{|l|c|c|c|c|}
\hline $\mathrm{FP}^{\mathrm{II}}$ & $g_{1}^{*}$ & $g_{2}^{*}$ & $u_{2}^{*}$ & $a^{\prime *}$ \\
\hline $\mathrm{FP}_{1}^{\mathrm{II}}$ & 0 & 0 & $\mathrm{NF}$ & $\mathrm{NF}$ \\
\hline $\mathrm{FP}_{2}^{\mathrm{II}}$ & 0 & $\frac{2 \varepsilon}{3}$ & 0 & 0 \\
\hline $\mathrm{FP}_{3}^{\mathrm{II}}$ & $\frac{2 y}{9}(3-\alpha)$ & 0 & $\frac{\alpha}{2(\alpha-3)}$ & 0 \\
\hline $\mathrm{FP}_{4}^{\mathrm{II}}$ & $\frac{2(\varepsilon-y)}{2 \alpha-9}$ & $\frac{4[3 \varepsilon+2 y(\alpha-6)]}{2 \alpha-9}$ & 1 & $\frac{\varepsilon(12-\alpha)+5 y(\alpha-6)}{\alpha(\varepsilon-y)}$ \\
\hline $\mathrm{FP}_{5}^{\mathrm{II}}$ & $-\frac{2[6 \varepsilon+5 y(\alpha-3)]}{3(9+\alpha)}$ & 0 & $\frac{3[\varepsilon+y(\alpha-1)]}{6 \varepsilon+5 y(\alpha-3)}$ & $\frac{18 \varepsilon-(\alpha-6)(\alpha-3) y}{\alpha[6 \varepsilon+5(\alpha-3) y]}$ \\
\hline $\mathrm{FP}_{6}^{\mathrm{II}}$ & $\mathrm{NF}$ & 0 & $\mathrm{NF}$ & $\mathrm{NF}$ \\
\hline $\mathrm{FP}_{7}^{\mathrm{II}}$ & $g_{1}^{*}$ & $g_{2}^{*}$ & $u_{2}^{*}$ & 0 \\
\hline $\mathrm{FP}_{8}^{\mathrm{II}}$ & $g_{1}^{*}$ & $g_{2}^{*}$ & $u_{2}^{*}$ & 0 \\
\hline
\end{tabular}

Table IV. List of all fixed points obtained in the frozen velocity limit. The value of the charge $u_{1}^{*}$ is equal to 0 for all points.

\begin{tabular}{|c|c|c|c|c|c|}
\hline $\mathrm{FP}$ & $g_{1}^{*}$ & $g_{2}^{*}$ & $u_{1}^{*}$ & $u_{2}^{*}$ & $a^{\prime *}$ \\
\hline $\mathrm{FP}_{7}^{\mathrm{II}}$ & 0.532193 & 9.89135 & 0 & 0.37859 & 0 \\
\hline $\mathrm{FP}_{1}^{\mathrm{III}}$ & 0.365039 & 6.38225 & 0.24709 & 0.352422 & 0 \\
\hline $\mathrm{FP}_{2}^{\mathrm{III}}$ & 0.399062 & 7.29847 & 0.148951 & 0.35954 & 0 \\
\hline
\end{tabular}

Table V. Coordinates of the IR stable fixed points obtained by numerical integration of $[39$ for $\alpha=110$ and $\varepsilon=1$ in the Kolmogorov regime $y=2 \eta=8 / 3$.

\begin{tabular}{|c|c|c|c|c|c|}
\hline $\mathrm{FP}$ & $g_{1}^{*}$ & $g_{2}^{*}$ & $u_{1}^{*}$ & $u_{2}^{*}$ & $a^{\prime *}$ \\
\hline $\mathrm{FP}_{7}^{\mathrm{II}}$ & 0.495405 & 9.92036 & 0 & 0.374461 & 0 \\
\hline $\mathrm{FP}_{1}^{\mathrm{III}}$ & 0.318124 & 6.0435 & 0.32542 & 0.339525 & 0 \\
\hline $\mathrm{FP}_{2}^{\mathrm{III}}$ & 0.381096 & 7.75271 & 0.121274 & 0.356122 & 0 \\
\hline
\end{tabular}

Table VI. Coordinates of the IR stable fixed points obtained by numerical integration of $(39)$ for $\alpha=110$ and $\varepsilon=2$ in the Kolmogorov regime $y=2 \eta=8 / 3$. 


\begin{tabular}{|c|c|c|c|}
\hline $\mathrm{FP}$ & $N(t)$ & $P(t)$ & $R^{2}(t)$ \\
\hline $\mathrm{FP}_{1}^{\mathrm{I}}$ & 0 & $\frac{\varepsilon}{4}-1$ & 1 \\
\hline $\mathrm{FP}_{2}^{\mathrm{I}}$ & $\frac{2 \varepsilon}{24-\varepsilon}$ & $\frac{7 \varepsilon-24}{24-\varepsilon}$ & $\frac{24}{24-\varepsilon}$ \\
\hline $\mathrm{FP}_{5}^{\mathrm{I}}$ & $\frac{(3+\alpha) \varepsilon-6 \xi}{3(5+2 \alpha)(2-\xi)}$ & $\frac{(18+7 \alpha) \varepsilon-12(5+2 \alpha)-6 \xi}{6(5+2 \alpha)(2-\xi)}$ & $\frac{2}{2-\xi}$ \\
\hline $\mathrm{FP}_{6}^{\mathrm{I}}$ & $\frac{2}{3} \frac{(3+\alpha) \varepsilon-6 \xi}{4(9+\alpha)-3(3+\alpha) \varepsilon+2(5+2 \alpha) \xi}$ & $\frac{3(\alpha+4) \varepsilon-4(9+2 \alpha)-6 \xi}{3[4(9+\alpha)-3(3+\alpha) \varepsilon+2(5+2 \alpha) \xi]}$ & $\frac{4(9+2 \alpha)}{4(9+2 \alpha)-3(3+\alpha) \varepsilon+2(5+2 \alpha) \xi}$ \\
\hline $\mathrm{FP}_{1}^{\mathrm{II}}$ & 0 & $\frac{\varepsilon}{4}-1$ & 1 \\
\hline $\mathrm{FP}_{2}^{\mathrm{II}}$ & $\frac{2 \varepsilon}{24-\varepsilon}$ & $\frac{7 \varepsilon-24}{24-\varepsilon}$ & $\frac{24}{24-\varepsilon}$ \\
\hline
\end{tabular}

Table VII. Analytical expressions for the given exponents of the Green functions (47). The corresponding expressions for FPs $\mathrm{FP}_{7}^{\mathrm{II}}, \mathrm{FP}_{1}^{\mathrm{III}}$ and $\mathrm{FP}_{2}^{\mathrm{III}}$ are not included, because it is not possible to determine their coordinates as explicit functions of free parameters of the model, i.e., as $(\varepsilon, y, \eta, \alpha)$.

[1] B. Schmittmann and R. K. P. Zia, Statistical Mechanics of Driven Diffusive Systems (Phase Transitions and Critical Phenomena vol 17) (ed C. Domb and J. L. Lebowitz, London:Academic, 1995).

[2] M. Henkel, H. Hinrichsen, and S. Lübeck, Nonequilibrium phase transitions: Volume 1 - Absorbing phase transitions (Springer, Dordrecht, 2008).

[3] U. Täuber, Critical Dynamics: A Field Theory Approach to Equilibrium and Non-Equilibrium Scaling Behavior (Cambridge University Press, New York, 2014).

[4] D. Stauffer and A. Aharony, Introduction to Percolation Theory (Taylor and Francis, London, 1992).

[5] H. K. Janssen, Z. Phys. B: Condens. Matter 42, 151 (1981).

[6] P. Grassberger, Z. Phys. B: Condens. Matter 47, 365 (1982).

[7] G. Ódor, Rev. Mod. Phys. 76, 663 (2004).

[8] H. K. Janssen and U. C. Täuber, Ann. Phys. 315, 147 (2004).

[9] J. L. Cardy and R. L. Sugar, J. Phys. A Math. Gen. 13, L423 (1980).

[10] P. C. Hohenberg and B. I. Halperin, Rev. Mod. Phys. 49, 435 (1977).

[11] P. Rupp, R. Richter, and I. Rehberg, Phys. Rev. E 67, 036209 (2003).

[12] K. A. Takeuchi, M. Kuroda, H. Chate, and M. Sano, Phys. Rev. Lett. 99, 234503 (2007).

[13] H. K. Janssen, K. Oerding, F. van Wijland, and H. J. Hilhorst, Eur. Phys. J. B 7, 137 (1999).

[14] H. Hinrichsen, Physica A 369, 1 (2006).

[15] H. Hinrichsen, J. Stat. Mech.: Theor. Exp. 2007, P07066 (2007).

[16] H. Hinrichsen, Adv. Phys. 49, 815 (2001).

[17] N. Sarkar, Phys. Rev. E 92, 042110 (2015).

[18] N. Sarkar and A. Basu, J. Stat. Mech.: Theor. Exp. 2014, P08016 (2014).

[19] H. K. Janssen, Phys. Rev. E 55, 6253 (1997).

[20] A. G. Moreira and R. Dickman, Phys. Rev. E 54, R3090 (1996).

[21] R. Cafiero, A. Gabrielli, and M. A. Muñoz, Phys. Rev. E 57, 5060 (1998).
[22] T. Vojta and M. Dickison, Phys. Rev. E 72, 036126 (2005).

[23] T. Vojta and M. Y. Lee, Phys. Rev. Lett. 96, 035701 (2006).

[24] L. D. Landau and E. M. Lifshitz, Fluid Mechanics (Pergamon Press, 1959).

[25] U. Frisch, Turbulence: The Legacy of A. N. Kolmogorov (Cambridge University Press, Cambridge, 1995).

[26] A. S. Monin and A. M. Yaglom, Statistical Fluid Mechanics:Vol 2 (MIT Press, Cambride, 1975).

[27] R. H. Kraichnan, Phys. Fluids 11, 945 (1968).

[28] N. V. Antonov, Phys. Rev. E 60, 6691 (1999).

[29] G. Falkovich, K. Gawȩdzki, and M. Vergassola, Rev. Mod. Phys. 73, 913 (2001).

[30] L. T. Adzhemyan, N. V. Antonov, and A. N. Vasil'ev, The Field Theoretic Renormalization Group in Fully Developed Turbulence (Gordon \& Breach, London, 1999).

[31] R. Benzi and D. R.Nelson, Physica D 238, 2003 (2009).

[32] S. Pigolotti, R. Benzi, M. H. Jensen, and D. R. Nelson, Phys. Rev. Lett. 108, 128102 (2012).

[33] R. Volk, C. Mauger, M. Bourgoin, C. Cottin-Bizonne, C. Ybert, and F. Raynal, Phys. Rev. E 90, 013027 (2014).

[34] M. DePietro, M. A. T. van Hinsberg, L. Biferale, H. J. H. Clercx, P. Perlekar, and F. Toschi, Phys. Rev. E 91, 053002 (2015).

[35] L. T. Adzhemyan and N. V. Antonov, Phys. Rev. E 58, 7381 (1998).

[36] N. V. Antonov, Physica D 144, 370 (2000).

[37] D. J. Amit and V. Martín-Mayor, Field Theory, the Renormalization Group and Critical Phenomena (World Scientific, Singapore, 2005).

[38] J. Zinn-Justin, Quantum Field Theory and Critical Phenomena (Oxford University Press, Oxford, 1996).

[39] A. N. Vasil'ev, The Field Theoretic Renormalization Group in Critical Behavior Theory and Stochastic Dynamics (Boca Raton: Chapman Hall/CRC, 2004).

[40] N. V. Antonov and A. S. Kapustin, J. Phys. A: Math. Theor. 43, 405001 (2010).

[41] N. V. Antonov, V. I. Iglovikov, and A. S. Kapustin, J. Phys. A: Math. Theor. 42, 135001 (2008).

[42] N. V. Antonov, A. S. Kapustin, and A. V. Malyshev, 
Theor. Math. Phys. 169, 1470 (2011).

[43] N. Sarkar and A. Basu, Phys. Rev. E 86, 021122 (2012).

[44] M. Dančo, M. Hnatič, T. Lučivjanský, and L. Mižišin, Theor. Math. Phys. 176, 898 (2013).

[45] N. V. Antonov, J. Phys. A 39, 7825 (2006).

[46] J. Honkonen and E. Karjalainen, J. Phys. A: Math. Gen. 21, 4217 (1988).

[47] J. P. Bouchaud and A. Georges, Phys. Rep. 195, 127 (1990).

[48] H. K. Janssen, Z. Phys. B 23, 377 (1976).

[49] C. de Dominicis, J. Phys. Colloq. France 37, C1 (1976).

[50] H. K. Janssen, Dynamical Critical Phenomena and Related Topics, Lect. Notes Phys. Vol. 104 (Springer, Heidelberg, 1979).

[51] P. Martin, E. D. Siggia, and H. A. Rose, Phys. Rev. A 8, 423 (1973).

[52] K. G. Wilson and J. Kogut, Phys. Rep. 12, 75 (1974).

[53] L. T. Adzhemyan, N. V. Antonov, and J. Honkonen, Phys. Rev. E 66, 036313 (2002).

[54] K. Symanzik, Lett. Nuovo Cimento 8, 771 (1973).

[55] R. Schloms and V. Dohm, Nucl. Phys. B 328, 639 (1989).

[56] Wolfram Research, Mathematica, Version 9.0 (Cham- paign, Illinois, 2012).

[57] D. Forster, D. R. Nelson, and M. J. Stephen, Phys. Rev. A 16, 732 (1977).

[58] M. Hnatich and J. Honkonen, Phys. Rev. E 61, 3904 (2000).

[59] M. Hnatič, J. Honkonen, and T. Lučivjanský, Eur. Phys. J. B 86, 214 (2013).

[60] M. Hnatich, J. Honkonen, and M. Jurcisin, Phys. Rev. E 64, 056411 (2001).

[61] D. Y. Volchenkov and M. Y. Nalimov, Theor. Math. Phys. 106, 375 (1996).

[62] N. V. Antonov, M. Y. Nalimov, and A. A. Udalov, Theor. Math. Phys. 110, 385 (1997).

[63] N. V. Antonov and M. M. Kostenko, Phys. Rev. E 90, 063016 (2014).

[64] C. W. Gardiner, Handbook of Stochastic Methods: For Physics, Chemistry, and the Natural Sciences (Springer, 2009).

[65] N. G. van Kampen, Stochastic processes in Physics and Chemistry (North-Holland, Amsterdam, 2007). 